\title{
GC-MS Analysis of Phyto-Chemical Constituents in Aqueous, Methanol and Ethyl Acetate Extracts of Vitis Vinifera Peel and Its Characterization Studies by Using AGNPs
}

\author{
${ }^{1}$ R. Divya, ${ }^{2 *}$. Supraja and ${ }^{1}$ E. David \\ ${ }^{1}$ Department of Biotechnology, Thiruvalluvar University, Serkkadu, Vellore- \\ 632001, Tamilnadu, India. \\ 2Nanotechnology Laboratory, Acharya N G Ranga Agricultural University, \\ Institute of Frontier Technology, Tirupati-517502, Andhra Pradesh, India.
}

Received: 24 Oct 2020 / Accepted: 22 Nov 2020/ Published online: 01 Jan 2021 *Corresponding Author Email: krishna.supraja@gmail.com

\begin{abstract}
Vitis Vinifera peel (Red) has major components such as Organic acids, Malate, higher moisture content and it is the source of medicine with high antioxidant activity for hundreds of years, Vitis vinifera belongs to family Vitaceae. But in our present work is designed to identify the possible phyto-chemicals compounds present in the Aqueous extracts, methanolic extract, and Ethyl acetate extracts of of Vitis vinifera peel by using GC-MS spectrum with their retention times indicating the peaks. This Vitis vinifera peel were shown nearly Thirty to Forty compounds from the Aqueous, Methanol and Ethyl acetate extracts the major chemical compounds were Xylose, Cyclo-hexanidiol, Octane, Butanoic acids and Octa-deca-diyndioic acids etc and from the Aqueous extracts we synthesized the AgNPs for preliminary characterization studies such as UV, DLS and SEM analysis.
\end{abstract}

Keywords

Vitis vinifera peel, AgNPs, Aqueous extract, Methanolic extract, Ethyl acetate extract, GC-MS spectrum.

\section{INTRODUCTION:}

Vitis vinifera is a member of the Vitaceae family, native to the Mediterranean region, central Europe and southwest Asia and cultivated today in all temperature regions of the world [1]. Vitis vinifera is used in conditions like hemorrhages, anemia, leprosy, skin diseases, syphilis, asthma, jaundice, bronchitis, anti-inflammatory, anti-carcinogenic, platelet aggregation inhibiting, and metal chelating properties [2-4]. Red Grapes or Vitis vinifera is a Berry fruit and belongs to the group of versatile fruits which are used in a wide range as popular foods from raisins to jelly to wine. Over 72 million tons of grapes are grown every year and 7.2 trillion gallons of wine is produced. Grapes are rich source of many vital nutrients and antioxidants. [5] V.vinifera seed contains lipid, protein, carbohydrates and 5-8\% polyphenols. The pulp majorly contains water (65\%- 
$85 \%$ of grape weight), sugars (15\%-25\%), it directs the sweetness. It is attributed to different mix of acids such as malic acid, tartaric acid, succinic acid, ascorbic acid, citric acid, phenols, and flavonoids. [6] These are considered to have biological properties, not only limited to antioxidant, anti-inflammatory, anti-cancer, antimicrobial, antiviral, cardioprotective, neuroprotective, hepatoprotective activities but also as a nutraceutical [7].

GC-MS is a method that couples two different analytical techniques, gas-liquid chromatography, and mass spectrometry to identify different phytochemical compounds present in a test sample. GC can separate volatile as well as semi-volatile compounds with higher resolution, but it cannot identify them. MS can be used to obtain structural information of the compound, but it cannot separate readily. It is used to analyze complex biochemical and organic mixtures and it is also highly compatible. [8] In addition, the size of the peaks determined by the spectrum is a directly proportional to the amount of material present in the test sample.

Several studies have indicated that extracts obtained from grape seed inhibit enzyme systems that are responsible for the production of free radicals, and that they have anti-mutagenic and anti-carcinogenic. It has a protective effect on oxidant-induced production and deposition of extracellular matrix components [9].

Hence the objective of the present study was aimed to identify the possible Phyto-chemical compounds using GC-MS Aqueous, Methanol and Ethyl acetate extracts of Vitis vinifera peel with the aid of GC-MS Technique and the AgNPs were synthesized by using the aqueous extracts of peel for UV, DLS and SEM analysis.

\section{MATERIALS AND METHODS:}

2.1. Preparation of Vitis vinifera peel aqueous extract.

The cleaned and air dried Vitis vinifera peels was taken and it was grounded by mortar and pestle until it turns in to thin fine powder then the powder was stored in refrigerator at 4 으 for further analysis. $10 \mathrm{~g}$ of Vitis vinifera powder was taken and dissolved in $100 \mathrm{ml}$ of distilled water and heated at $80^{\circ} \mathrm{C}$ for $1 \mathrm{~h}$ then the extract was filtered by using Whatman No. 1 filter paper and collected in plastic bottle and stored at $4 \pm C$ for further characterization and experimentation [10-11].

2.2. Preparation of Vitis vinifera peel extract mediated silver $(\mathrm{Ag})$ nanoparticles.

Silver nitrate (>99\% pure) was purchased from Sigma- Aldrich, India. To prepare the AgNPs, a $90-\mathrm{mL}$ aqueous solution of $1.0 \times 10^{-3} \mathrm{M}$ silver nitrate was mixed with a $10-\mathrm{mL}$ of $5 \%$ aqueous solution of vitis vinifera peel extract. The vitis vinifera $\mathrm{Ag}$ solution was yellow in color and the solution was stirred repeatedly for an hour, and it was observed that the color of the solution has been changed to brown which visually confirms the formation of Nanoparticles. These vitis vinifera silver Nanoparticles were characterized by using the techniques such as UV-Vis spectrophotometry, Dynamic light scattering (Particle size), zeta potential and Scanning electron microscopy (SEM) analysis [12].

\subsection{Vitis vinifera peel sample extractions}

Twenty grams of the powdered peels were extracted with $100 \mathrm{~mL}$ of $40 \%$ methanol overnight in a stopped bottle and with occasional stirring at room temperature $\left(28^{\circ} \mathrm{C}\right)$. The sample was first sieved using muslin cloth and then filtered using Whatman No.1 filter paper. This process was repeated three times to remove the contaminants in sample. The filtrate was concentrated under rotary vacuum evaporator for one hour at $50^{\circ} \mathrm{C}$ and then lyophilized to get a compounds extract. The dry extract was preserved under 4 으 until further use and the same procedure used for ethyl acetate compound extraction [13].

2.4. Gas chromatography-mass spectrometry (GCMS) and identification of phytocompounds

GC-MS analysis was carried out on a GC-MS (Model: QP2010 PLUS Shimadzu, Japan) comprising a AOC-20i auto-sampler and chromatograph interfaced to a mass spectrometer (GC-MS). The instrument was equipped with a VF 5 ms fused silica capillary column of $30 \mathrm{~m}$ length, $0.25 \mathrm{~mm}$ diameter and $0.25 \mu \mathrm{m}$ film thickness. The temperatures employed were column oven temperature $80^{\circ} \mathrm{C}$, Injection Temp $250^{\circ} \mathrm{C}$ at a pressure of $108.0 \mathrm{kPa}$, scan range $40-800 \mathrm{u}$ and an injection volume of $1 \mu \mathrm{L}$ of the plant extract (split ratio 10:1). The total running time of GC-MS was 30 min. The relative percentage of the extract was expressed as percentage with peak area normalization. The fragmentation pattern spectra of the unknown components were compared with those of known components stored in the NISTV.3.2 library [14]. The compound bioactivity prediction was based on Dr. Dukes Phytochemical and Ethnobotanical Databases. The name, molecular weight, and structure of the components of the test materials were ascertained [15].

\section{RESULTA AND DISCUSSION:}

3.1. UV-visible spectrum of Vitis vinifera Peel aqueous extract mediated synthesized AgNPs

In this study, extract when interacting with the silver nitrate salt solution form a dark brown solution due 
to the reduction of the silver ion to AgNPs followed by a colour change indicating the biotransformation of ionic silver to reduced silver and the subsequent formation of AgNPs in an aqueous medium. The colour change was monitored visually and the peak at $316 \mathrm{~nm}$ in the UV-visible spectra indicated the presence of AgNPs which may be due to the excitation of surface plasmon resonance vibrations in AgNPs (Figure 1) [16].

3.2. Dynamic light scattering analysis of Vitis vinifera Peel aqueous extract mediated synthesized AgNPs

The hydrodynamic diameter (size) of the AgNPs was found to be $87.6 \mathrm{~nm}$ (Figure 2a) and was measured as a function of scattering angle of the laser from the surface of the particle. Further, zeta potential of AgNPs was also measured and was recorded as $48.7 \mathrm{mV}$ (Figure 2b). The zeta potential clearly indicates stability of the prepared AgNPs [17].

3.3. Scanning electron microscopic analysis of Vitis vinifera Peel aqueous extract mediated synthesized AgNPs

Surface morphology of silver nanoparticles was studied from the SEM micrograph. It is evident that AgNPs were spherical, irregular in shape and some of them were poly dispersed. The measured average size of AgNPs was 30-50 $\mu$ m (Figure 3) $[12,18]$.

3.4. Identification of Phyto-compounds present in Aqueous, Methanolic and Ethyl acetate extractions from Vitis vinifera peel

3.4.1. GC-MS analysis of Aqueous extract of Vitis vinifera peel

GC-MS chromatogram of the aqueous extract of Vitis vinifera peel showed four peaks (Figure. 4) in chromatogram indicating the presence of four phytochemical constituents. On comparison of the mass spectra of the constituents with the NIST08, WILEY8 and FAME libraries the four phyto-constituents were characterized and identified in (Figure 5)

The retention time present at $\mathbf{2 . 8 6}$ each showing the compound of amphetamine-3-methyl.

The retention time at $\mathbf{1 9 . 7 4}$ each showing the compounds of Xylose,1-P-Toluidino-1-Deoxy-Beta-DIdopyranose,2-1,2-Dihydroxyethyl-9-Beta-DRibofuranosylHypoxanthine,Methyl-Alpha-D-

Ribofuranoside, He xanoicacid,Hexanoicacid,6Bromo,9-Bromononanoicacid,D-Allose,Allo-

Inositol,Ribonicacid.Gamma-Lactone, 1,2,5,6-Di-OIsopropylidene-3-o-

Methanesulfonyl,glucofurnose,Beta-D-

Glucopyranose,1,6-Anhydro,5-Thio-D-

Glucopyranose,1,6-Anhydro-Beta-D-

Talopyranose,D-Galactose,6-Deoxy, Lactose,Beta-

Glucopyranose,1,6-Anhydro,5-Cyano-
Desoxinojirimycin,D-Chiro-Inositol,3-o-2-Amini-4Carboxyminomethylamino-2,3,4,6-TE.

The retention time at $\mathbf{2 6 . 0 5}$ showing the compounds of 1, 3-Cyclohexanediol, 1, 3-Cyclohexanediol,CisEthylamine,1-Methyl-2-5-Methyl-1H-Pyrazol-3-yl-, 1, 3-Cyclohexanediol,Trans-1H-Imidazole-4-

Ethanamine,n, 5-Dimethyl-, 4H-1, 3, 4-Triazol-3Amine, N-Dimethyl amino methylene-, Ethyl2-2Chloroacetamido-3,3,3-Trifluor olactate, $1 \mathrm{H}$ -

Pyrazole,1-Methyl-4-Methylaminomethyl-,1HPyrazole,1-Methyl-4-Methylaminomethyl-,1-Beta D-Ribofuranosyl-s-Triazol(2,3-A-S-Triazin-5,7-

Dione, Ribavirin, Glucopyranuronamide,1-4-Amino-2oxo-1(2H) -Pyrmidinyl-1-4-Dideoxy,1H-Imidazole,2Ethyl-4,5-Dihydro-,Histamie,N-Triflouroacetyl-2-

Amino-,Cyclohexan e-3-5-diol,Cis-,2Hydroxymethylcyclopentanol, $1 \mathrm{H}, 3 \mathrm{H}-$

Furo(3,4Furan,Tetrahydro-,1H-Imidazole,2-Ethyl4,5-Dihydro-4-Methyl.

The retention time at $\mathbf{2 6 . 5 8}$ showing the compounds 22, 23-Dibromostigmasterolacetate, 1-Hydroxy-1, 7Dimethyl-4-Isopropyl-2, 7-Cyclodecadiene, 10-12Pentacosadiynoicacid, Methyl, 10, 12Pentacosadiyonate,5,8, 11,14-eicosatetraenoicacid, Methyl ester, All-Z-, Pregn-4-Ene-3,20-Dione,17,21dihydroxy-Bis-o-Methyloxime.

3.4.2. GC-MS analysis of Ethyl acetate extract of Vitis vinifera peel

GC-MS chromatogram of the ethyl acetate extract of Vitis vinifera peel showed thirteen peaks (Figure 6) in chromatogram indicating the presence of thirteen phyto-chemical constituents. On comparison of the mass spectra of the constituents with the NIST08, WILEY8 and FAME libraries the thirteen phytoconstituents were characterized and identified in (Figure7).

The retention time present at $\mathbf{2 . 8 5}$ each showing the compounds of S (-)- Cathionone, N-Acetyl, Acetamide, 2, 2, 2-Triflouro-, Acetamide, 222, Trifluro-, 2, 3-Pyridinecarboxylic acid.

The retention time present at $\mathbf{1 9 . 2 7}$ each showing the compounds of 1,3-Cyclohexanedioldiacetatecis-, 1, 4-CyclohexanediolDiacetateTrans-,1,3Cyclohexanediol diacetate Trans-, L-Alanine NAcetyl-3-Chloro-Methyl ester, 3-Cyclohexen-1-olAcetate, 4(H)-Pyridine N Acetyl-

The retention time present at $\mathbf{1 9 . 7 5}$ each showing the compounds of 2-Butyl-1-2-Oxaborolane, Ether Hexyl Pentyl, Dichloroacetic acid 4-Methyl Pentyl ester, Sulfurous acid Iso hexyl 2-Pentyl ester,6,8Doixatetradecane, 5ilsoxazolecarboxylix acid 4,5Dihydro-5-Methyl ester(R), Di(Tetrahydrofurfuryl) Adipate, Pyrrolidine 1,1-Methylenebis,2-Ethyl-1Butanol Triflouroacetate, sulphurous acid Hexyl Pentyl ester, Succinic acid Hexadecyl 
Tetrahydrofurfuryl ester, 1-Butanol 4Hexyloxy,Succinic acid Octadecyl Tetrahydrofurfuryl ester,2-Ethylbutyl Isobutyrate,Succinic acid Heptadecyl Tetrahydrofurfuryl ester, 1-Octacosanol 2,4,6,8 Tetramethyl-All-R,Sulfurous acid Isohexyl Pentyl ester, Sulfurous acid Nonyl 2-Propyl ester,Butanoic acid 2-Ethyl Butyl Ester, Dichloroacetic acid 6-Ethyl-3-Octyl ester.

The retention time present at $\mathbf{2 0 . 5 1}$ each showing the compounds of 2,5-Diamino-2-Methylpentanoic acid,4-cycloocten-q-Amine N Methyl,2-T Butylperoxy-2-Ethylbutan-1-ol Butyrate ester, Octane 1,1-Oxybis, Methoxyacetic acid octyl ester,Acetic acid Trichloro-Octyl ester,10Undecenoic acid octyl ester,butanoic acid octylester,2-Bromoproponoicacidoctylester,5-

Octadecenal,4-Amino-6-Methyl-Piperidin-2one,Chloromethyl octylether, $2,4(1 \mathrm{H}, 3 \mathrm{H})$ Pyridinedione1-Beta-D-Ribofuranosyl,Sulfurous acid Decyl 2-Propyl ester, Hexyl Octyl ether,2-Propenoic acid Pentadecyl ester,1,2,3-cyclohexanetriol,2Propenoic acid Tridecyl ester.

The retention time present at $\mathbf{2 1 . 2 3}$ each showing the compounds of Octadecane 2Methyl,Hexadecane, Nonadecane 2-Methyl,Eicosane 2-Methyl,Tricosane 2-Methyl,Tricosane2Methyl,Tricosane2-Methyl, Docosane2,21Dimethyl,Heptadecane2,6,10,14-

Tetramethyl,Hexadecane,1-lodo-2-

Methylundecane,Penta decane,Nonadecane2Methyl,Undecane2,10-Dimethyl,Heptadecane2-

Methyl,Hexadecane,Octadecane,1-

lodo,Tetradecane, Heptadecane,4-

Methyl,Pentadecane 2-Methyl,Decane 2,6,8Trimethyl.

The retention time present at $\mathbf{2 2 . 2 9}$ each showing the compounds of Tricyclo[4.4.0.0(3,8)Dec-9-En-4Ol,2,4Decadien1-

Ol(E,Z),9,10Dimethylenetricyclo[4.2.1.1(2,5)Decane, 1,2,4-Methenocyclobut[CD]Inden-3-Dione3A,4,7,7ATetrahydro-5-Methyl,4,7-Methanoisobenzofuran1,3-Dione,3A,4,7,7A-Tetrahydro-5-Methyl,1-2Methylenecyclohexyl-3-Phenylpropan-1-

OL,1,3,5Dodecatriene,6-[(1E)-1,3-Butadienyl-1,4Cycloheptadiene,4,7-Methanoisobenzofuran-1,3Dione3A,4,7,7A-Tetrahydromethyl,3-[(1Z)-1,3Butadienyl]-1-4-Vinylcyclopentene,6-[(1Z-1,3Butadienyl]-1,4-Cycloheptadiene,Cyclobutane,1(1,3-butadienyl-2-Vinyl,1,3,7,11-

Cyclotetradecatetraene,2,7-

Methanopthalene, 1,2,4A,7,8,8A-

Hexahydro,Cyclobutane1,2-Bis(1,3Butadienyl),1,2-

Bis(3-Cyclohexenyl)ethylene,1,4,4A,5,8,8A-

Hexahydronaphthalene, $1,2,4 \mathrm{a}, 4 \mathrm{~B}, 7,8,8 \mathrm{~A}, 8 \mathrm{~B}$ -
Octahydrobiphenylene,Tricyclo[4.3.0.0(3,7)]Non-8En-4-Ol.

The retention time present at $\mathbf{2 3 . 0 8}$ each showing the compounds of Methyl 5-2-Phenyl propionyl Hexanoate, 2-Methyl-1-Phenyl-2-Propen-1-Ol, Tricyclo [4.2.1.1(2, 5)DecA-3, 7-Dien-9-One 10Hydroxy-10-Methyl-Stere.

The retention time present at $\mathbf{2 6 . 5 4}$ each showing the compound of $1 \mathrm{H}-4-$ Azacycloprop [CD]Indene octahydro-4-Methyl-.

The retention time present at $\mathbf{3 0 . 5 8}$ each showing the compounds of Tricyclo[4.4.0.0(2,7)Dec-3-Ene-3Methanol,1-Methyl-8-1-Methyethyl,2,6-Dimethyl-4Nitro-3-Phenyl-Cyclohexanone,2,5-

Octadecadiynoicacid, methyl,Ester,10,12-

Docasadiyndiocacid,Falcarinol,10-Heneicosene11-

Phenyl,4,7-

OctadecadiynoicacidMethylester, Methyl5,7-

Hexadecadiyonate,Estra-5-(10)-En-3-One-17-

OLacetate,(4,4-Dimethyl-2,4,5,6-Tetrahydro-1H-

Inden-2-YL)Aceticacid,Methyl5,7-

Hexadecadiynoate,Estra-5(10)-En-3-One-17-

Olacetate,(4,4-Dimethyl-2,4,5,6-Tetrahydro-1H-

Inden-2-YL)Aceticacid, Methyl-8,10-

Octadecadiynoate,Methyl-7,9-Octadecadiynoate,1-

3,3-Dimethyl-1-YL-2,2-Dimethylcyclopropene-3-

Carboxylic acid [19].

3.4.3. GC-MS analysis of Methanol extract of Vitis vinifera peel

GC-MS chromatogram of the methanol extract of Vitis vinifera peel showed sixteen peaks (Figure 8 ) in chromatogram indicating the presence of sixteen phyto-chemical constituents. On comparison of the mass spectra of the constituents with the NIST08, WILEY8 and FAME libraries the sixteen phytoconstituents were characterized and identified in (Figure 9)

The retention time present at $\mathbf{9 . 5 8}$ each showing the compounds of 4-Methyl-1-3-Oxazine-2,6[3H]Dione,Dec-9-En-6-Oxo-1-Ylamide,1,2,5-Oxadiazole-

3-Carbohydroxymicacid,4-

Amino,4(1H)Pyrimidinone,6-Amino-2-Methyl-5nitroso-,Propanenitrile3-Amino-2-Methyl-5-Nitroso,Prpanenitrile,3-Amino-2,3-

Dihydroxymino, Glutamine, $\mathrm{N}-$ Methyl-Oxime, $3(2) \mathrm{H}$ -

Isoxazolone,4,5-Dimethyl,2,4(1H,3H)-

Pyrimidinededione,1-[3,5-Dihydroxy-6-

Hydroxymethyl],1,2,3-Triazole-4-Methanol,1-4-

Aminofurazan-3-YL),3-Methyl-3-5-

(Cyanoethyl)Tetrahydro-4-

Thiopyranone,Cycloheptano[D]Imidazolidine,1,3-

Dihydroxy-2-Methyl-,1H-Imidazole,2,4-Dimethyl-

,Ronidazole,Propanal,2-Methyl-Oxime,L-

Guanindinosuccinimide,5-Amino-3,4-Dimethyl-

Isoxazole,Spiro[Tetrahydrofuran-3,5'-Hydantoin,1,3- 
Oxazin-2-one, Tetrahydro-3-Methyl-5-6-

TrimethyleneCis-,1H-1,2,3-Triazole-5-Methanol,1-4Amino-1,2,5-Oxadiazole-3-YL)-

The retention time present at $\mathbf{1 2 . 2 7}$ each consisting compounds of 4-Chloro-1-Butanol,4-Chloro-1ButanolOxirane,Propyl-,Propylaldoxime,2-MethylAnti,3-Ethyl-3-Methyldiaziidine,4-

Hydroxybutylacrylate,4-Hydroxybutylacrylate,1,2,5Triazole-2-oxide,1-sec-Butyl-3-Nitro-4-Amino-,1Dimethylamino-2-Nitroethylene, L-Gala-L-IdoOctose,5-Amino-3,4-DimethylIsoxazole,Pentanoicacid,3-Hydroxy-4-MethylMethylester,Cycloheptanol,3,8,9-

Trioxabicyclo[4.2.1]nonane, aceticacid, Pentylester,C yclohexanol2-Methyl-Trans-,Isothiourea,2-2-

Octylsulfonyl ethyl-4-amino-1-Hexanol.

The retention time present at $\mathbf{1 4 . 4 7}$ each consisting the compounds of Furazanamine,4-Azido-,3Ethylamino-5-Hexene-2-OL.

The retention time present at $\mathbf{1 7 . 4 9}$ each consisting the compounds of Cyclopropaneteteradecanoicacid,2-octyl-methyl ester, 1,3,2,5-Diformal-L-Rhaminitol acetate,Dodecanoicacid,2,3-

bis(Acetyloxy)Propylester,Lyxitol,1-0-nonyl,2-

Butanyl,3-1,3-Dimethylbutoxy-3-Pentanol-3-

Methyl,3-Pentanol-3-Methyl-,Alpha-D-

Glucopyranoside,Methyl3,6-Anhydro,Pyrimidine-

2,4,6(1H,3H,5H-Trione,1-Octadecyl-,3-Octanol,3,7Dimethyl-,3-Octanol,3,6-Dimethyl-,3-octanol,3,7-

Dimethyl-,2-Methyl-3-

Decanol,Eicosanoicacid,1,2,3,4-

Pentadecanetetrol,[2R-(2R,3s,4s)],1-Octanamine,N-

Methyl-N-Nitroso-,4-Heptanol,2,6-dimethyl-4-

Propyl,1,2,3,4-Hexadecanetetrol,2R-2R,3S,4S)],D-

Mannohexadecane-1,2-3,4,5-Pentol,Undecanol-4.

The retention time present at $\mathbf{1 8 . 4 2}$ each consisting

the compounds of 2-Pentenoicacid,4-oxo-

Methylester(z),Pentanoicacid,3,5-dioxo-

Methylester,Hexanoicacid,3-Oxo-Methylester,2-

Pentanoicacid,4-oxo-Methylester,(E),2(5H)-

Furanone,5,5-Dimethyl-,1,3-Dioxolane,2,2-

Dimethyl-4-Hydroxymethyl-5-2-

Hydroxypropyl,Xylopyranoside,Methyl4-Thio-

Triacetate,Alpha-D-,2(5H)-Furanone,5,5-

Dimethyl,1,3-Dixolane,2,2-Dimethyl-4-

Hydroxymethyl-5-2-

Hydroxypropyl,Xylopyranosidemethyl-4-Thio-

Triacetate,Alpha-D,2(5H)Furanone,5,5-Dimethyl-

,Heptanedioic acid 4-Methyl-Dimethyl ester, Acetic acid,2-Methyl-6-oxo-Heptyl ester,Hexanoicacid-5oxo-methyl ester, Heptanedioc acid 3-MethylDimethylester,Methyl-4-oxo-2-Pentenoate,5Isoxazolidinecarboxylicacid,5-Methyl-2-5-O-Methyl2-3-O-1-Methy,2-Propanol,1,3-Dimethylbutoxy,3-
Ethyl-4-Methyl-3

Furanhexanoicacid, Tetrahydro-

Beta,Delta,Dihydroxy-5-Metho,5-

Isoxazolidine, carboylicacid,5,Methyl-2-5-0-2-3-0-1-

Methylethyl,1,4-DI-O-Acetyl-2,5-DI-O-Methyl-3,6-

Dideoxy-D-Glucitol.

The retention time present at $\mathbf{1 8 . 2 7}$ each consisting the compounds of 5-Hexenoicacid Methylester,3Dodecen-1-OL,2-Heptadecenal,4-

OctadecenoicacidMethylester, Undecanenitrile,4chloro-3-N-Hexyltetrahydropyran, Hexanenitrile,5Methyl-,Decanitrile,Methyl-3-

Cyclopropylpropanoate,Nonanenitrile,E-2-

Octadecen-1-OL,Undecanenitrile,Z-3-Octadecen-1-

OL-Acetate,5-Hexanoic,acid, methylester,1,19-

Eicosadiene,z-2-Octadecen-1-

OL,Acetate,Decanenitrile,Cyclohexanol-2-Methyl-

AcetateCis,Oxalicacid Cyclohexyl methyl propyl ester,2-Dodecen-1-OL.

The retention time present at $\mathbf{2 0 . 0 4}$ each consisting the compound of 2-Propenoicacid,3-2-2-Dimethyl-13-Dioxolan-4-YL-Methyl ester(s)-

The retention time present at $\mathbf{2 2 . 5 6}$ each consisting the compounds of Butane,1,1'Ethylidennebis(Oxy)Bis-2-Methyl,1,6-Heptadien-4-

OL,9-Octadecen-12-ynoic acid Methyl ester,5Dimethylamino-2-Methyl-4-

Oxazolecarbonitrile,Disulphide Isopentyl methyl,8,11,14-Eicosatrienoic acid Methyl ester(Z,Z,Z)-, Butanoyl chloride, Methyl Pentyl disulfide,5-Methoxy-cyclooctene,Bicyclo[2.2.2]Oct-

5-En-2 Y-Ldimethylamine,1,4-dioxane 2,6Dimethyl,2,6-Dimethylmorpholine-4-Carbothioic acid,2-1-2-Pyrimidyl]Ethynide.

The retention time present at $\mathbf{2 4 . 6 3}$ each consisting the compounds of Cyclohexane,2,4-Disopropyl-1,1Dimethyl, Cyclohexane,1,5-Disopropyl-2,3-Dimethyl, Cyclohexane,1,1'-1 Methyl propylidene Bis, Oxalic acid Heptadecylic 1-Menthyl ester, Oxalic acid 1Menthyl pentadactyl ester.

The retention time present at $\mathbf{2 6 . 4 1}$ each consisting the compounds of 1-Hexanol,2-Hydroxymethyl, AminopropionamideN-Methyl-N-4-1-Pyrrolidinyl-2Butynyl-N-T-But2-Octenal(E),3-Methyl-4-Phenylthio2-Prop-2-enyl-2,5-Dihydrothiophene 1,1-Dioxi, Fromamide N-Methyl-N-4-1-Pyrrolidinyl-2-Butynyl. [20-21], both worked on GC-MS of grape seeds and their results demonstrated 33 and 16 constituents respectively against the outcome of this study where 7 peaks were observed. Also, [22] in aqueous skin extract reported 16 compounds. Out of the compounds posited by other researchers only palmitic and stearic acid were in affirmation with the result. The disparity in the number and nature of compounds found in this research could be due to 
differences in plant parts, solvents of extraction and geographical location.

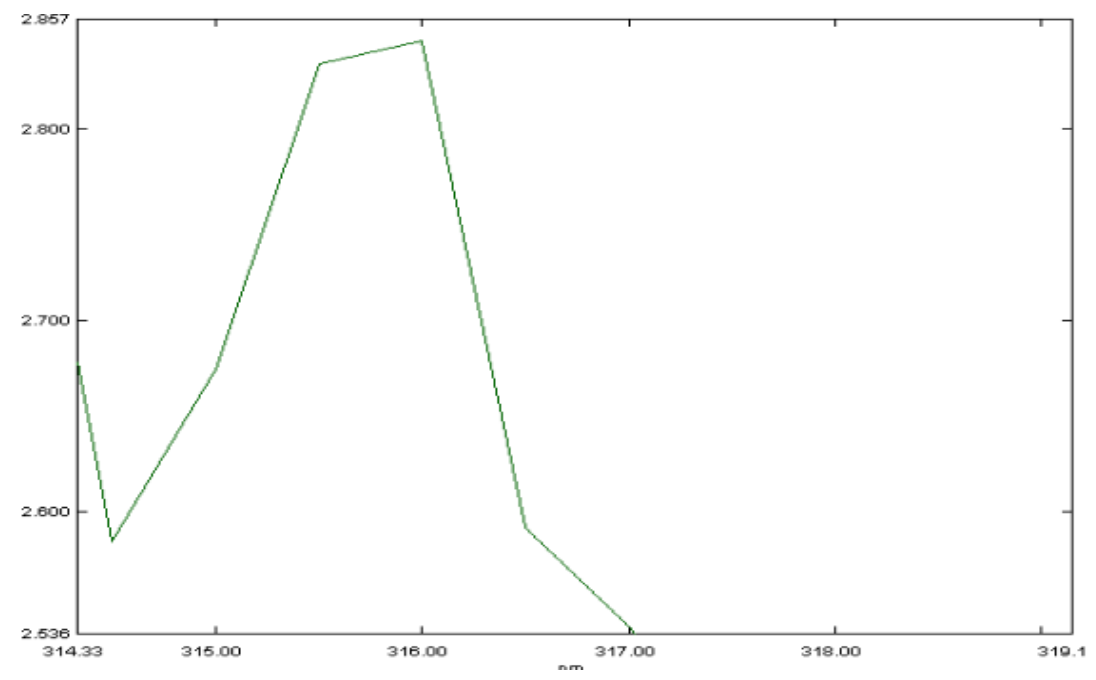

Figure.1 Showing UV-Visible spectrum analysis of Vitis vinifera peel extracted mediated synthesized Silver nanoparticles.
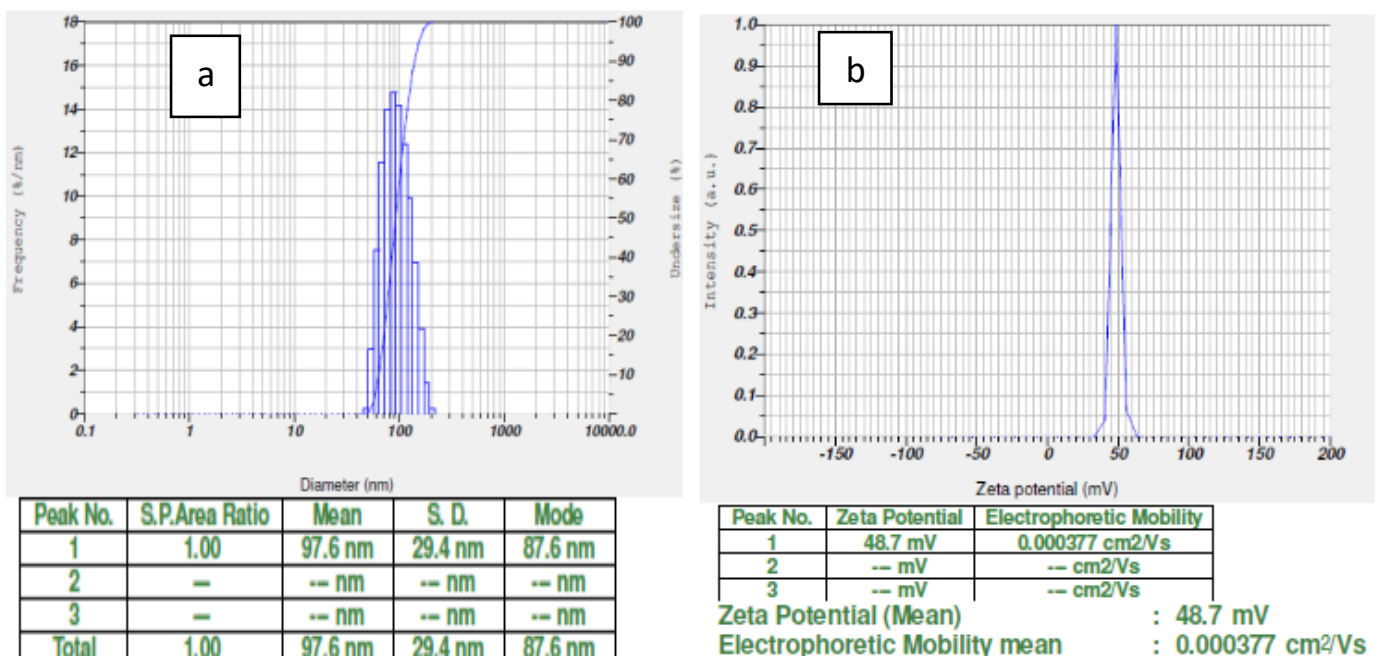

Figure.2 Showing Dynamic light scattering analysis of Vitis vinifera peel extracted mediated synthesized Silver nanoparticles a) Particle size b) Zeta potential.
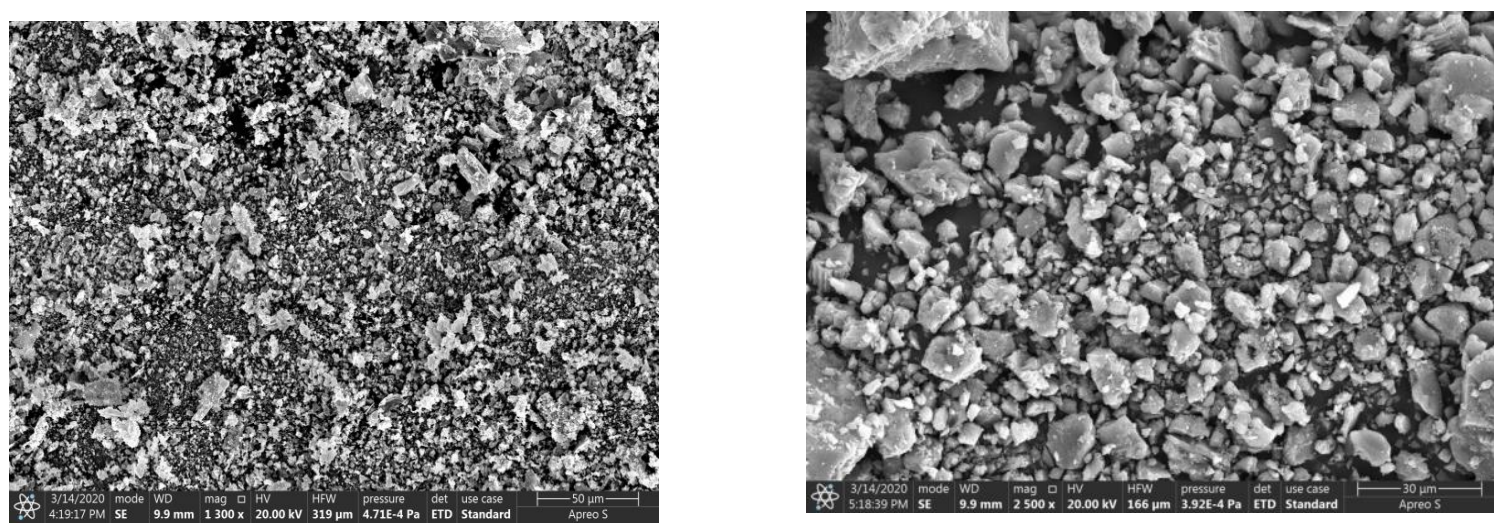

Figure.3 Showing Scanning electron microscopy analysis of Vitis vinifera peel extracted mediated synthesized Silver nanoparticles. 


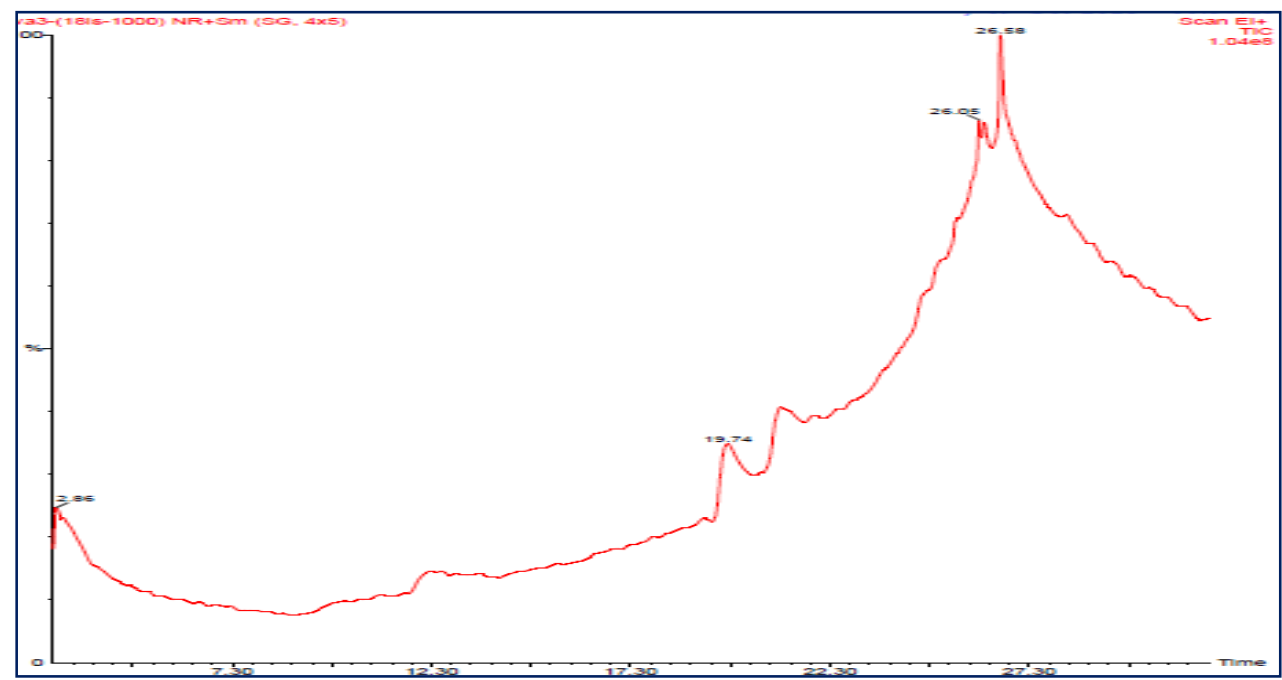

Figure.4 showing GC-MS Chromatogram of Aqueous extract of the Vitis vinifera peel
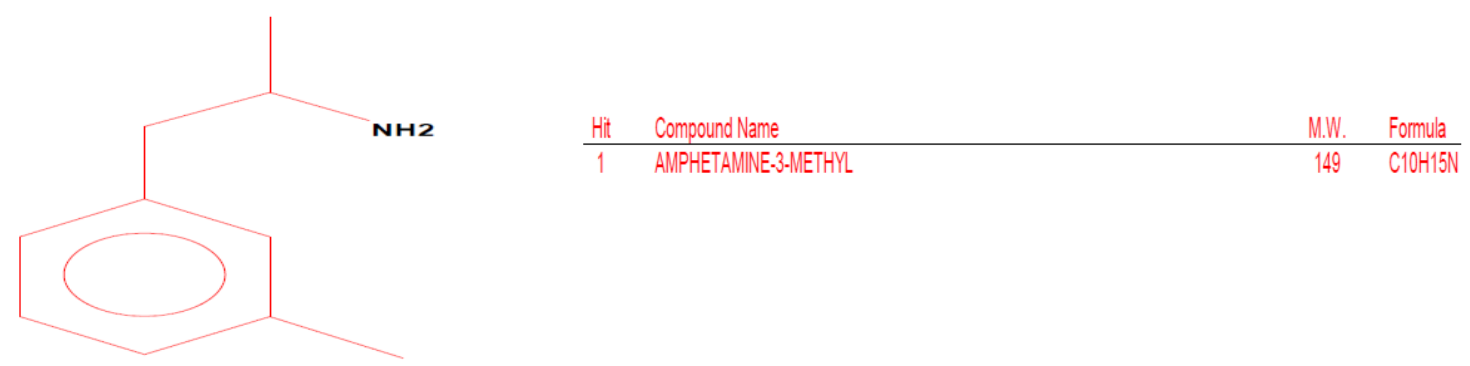

19.74

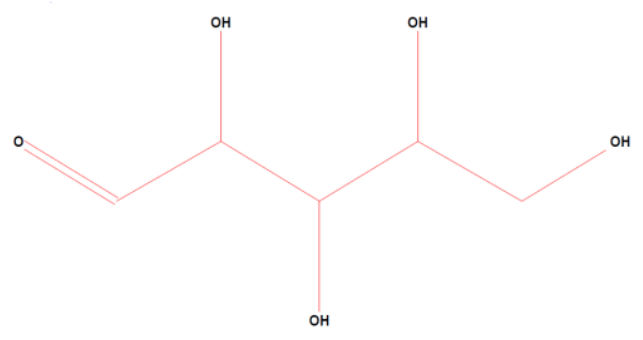

\begin{tabular}{|c|c|c|c|}
\hline Hit & Compound Name & M.W. & Formula \\
\hline 1 & XYLOSE & 150 & $\mathrm{C} 5 \mathrm{H} 1005$ \\
\hline & $\begin{array}{l}\text { 1-(P-TOLUIDINO)-1-DEOXY-BETA.-D-IDOPYRANOSE } \\
\text { 2-(1.2-DIHYDROXYETHYL-9-[BETA.-D-RIBOFURANOSYL]HYPOXANTHINE }\end{array}$ & $\begin{array}{l}269 \\
328\end{array}$ & $\begin{array}{l}\mathrm{C} 13 \mathrm{H} 1905 \mathrm{~N} \\
\mathrm{C} 12 \mathrm{H} 1607 \mathrm{~N} 4\end{array}$ \\
\hline 4 & 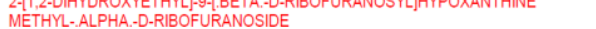 & 164 & $\mathrm{C} 6 \mathrm{H} 12 \mathrm{O} 1 \mathrm{~N}$ \\
\hline & HEXANOIC ACID & 116 & $\mathrm{C} 6 \mathrm{H} 12 \mathrm{O} 2$ \\
\hline & HEXANOIC ACID, 6-BROMO- & 194 & $\mathrm{C} 6 \mathrm{H} 1102 \mathrm{Br}$ \\
\hline & 9-BROMONONANOIC ACID & 236 & $\mathrm{C} 9 \mathrm{H} 1702 \mathrm{Br}$ \\
\hline & D-ALLOSE & 180 & $\mathrm{C} 6 \mathrm{H} 1206$ \\
\hline & ALLO-INOSITOL & 180 & $\mathrm{C} 6 \mathrm{H} 12 \mathrm{O} 6$ \\
\hline 10 & D-(+)-RIBONIC ACID .GAMMA.LACTONE & 148 & $\mathrm{C5H} 805$ \\
\hline & 1,2,5,6-DI-O-ISOPROPYLIDENE-3-O-METHANESULFONYL GLUCOFURANOSE & 338 & C13H2208S \\
\hline & HEXANOIC ACID, 6-BROMO- & 194 & $\mathrm{C} 6 \mathrm{H} 1102 \mathrm{Br}$ \\
\hline & BETA.D-GLUCOPYRANOSE, 1,6-ANHYDRO- & 162 & $\mathrm{C} 6 \mathrm{H} 1005$ \\
\hline & 5-THIO-D-GLUCOPYRANOSE & 196 & $\mathrm{C} 6 \mathrm{H} 1205 \mathrm{~S}$ \\
\hline & 1,6-ANHYDRO-BETA.D-TALOPYRANOSE & 162 & C6H1005 \\
\hline & D-GALACTOSE, 6-DEOXY- & & $\mathrm{C} 6 \mathrm{H} 1205$ \\
\hline 17 & LACTOSE & 342 & C12H22O11 \\
\hline 18 & BETA.D-GLUCOPYRANOSE, & 16 & $\mathrm{C} 6 \mathrm{H} 1005$ \\
\hline 19 & & & $\mathrm{C} 7 \mathrm{H} 12 \mathrm{ON} 2$ \\
\hline 20 & D-CHIRO-INOSITOL, 3-O--(2-AMINO-4-((CARBOXYIMINOMETHYL)AMINO)-2.3,4,6-TE & 379 & $\mathrm{C}_{44 \mathrm{H} 2509 \mathrm{~N} 3}$ \\
\hline
\end{tabular}

26.05

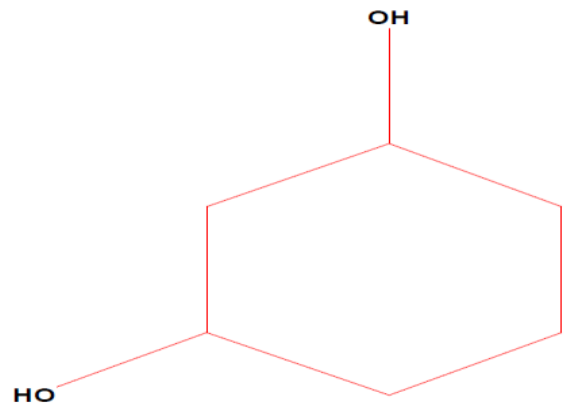

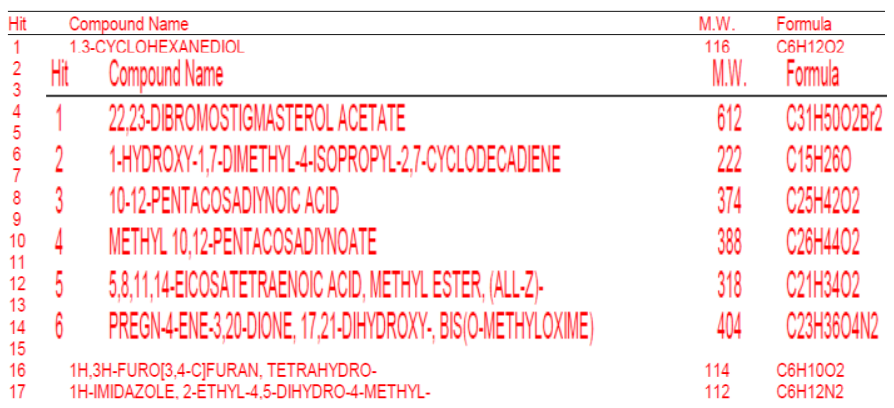

26.58 


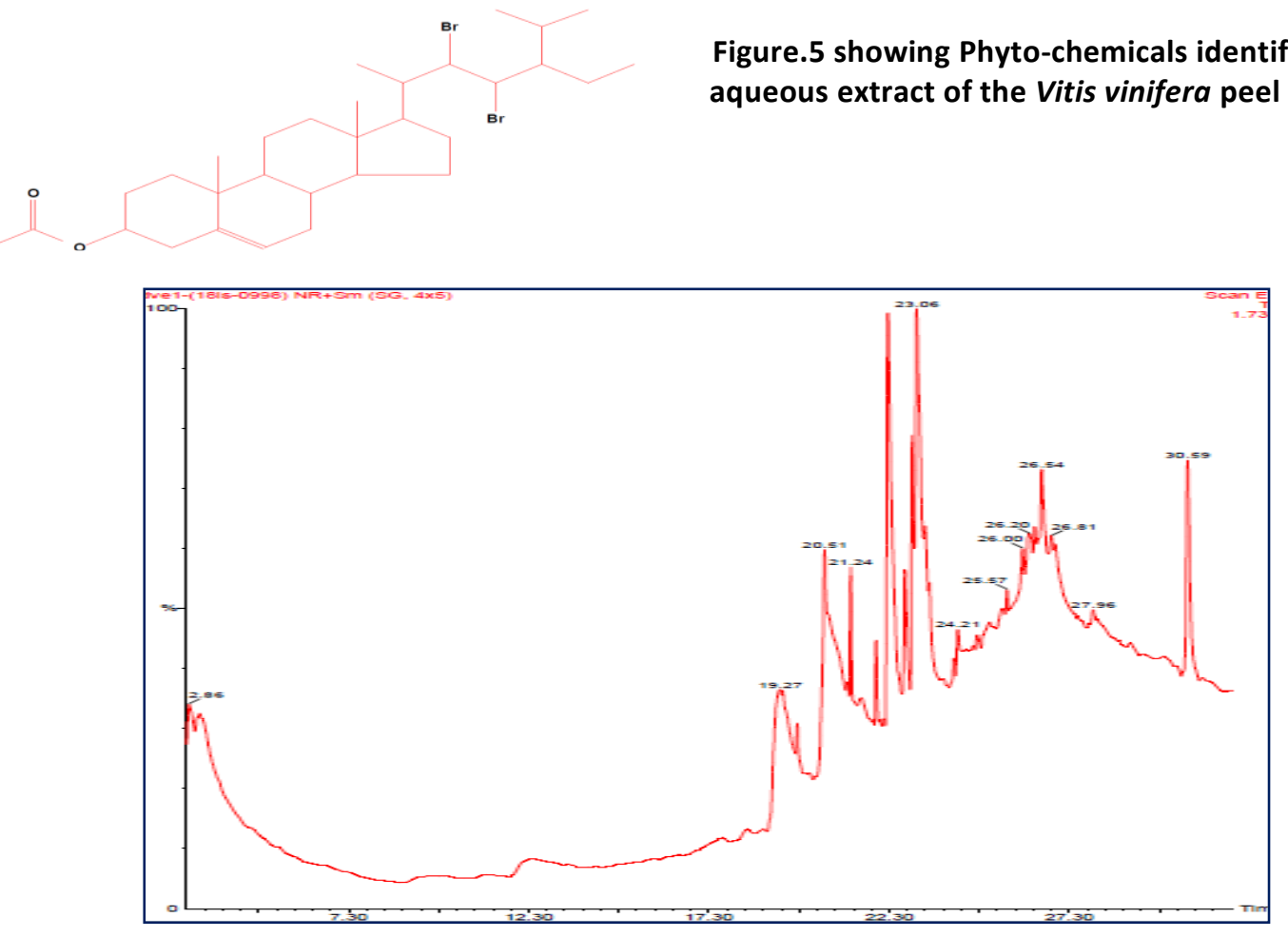

Figure.6 Showing GC-MS Chromatogram of Ethyl acetate extract of the Vitis vinifera peel

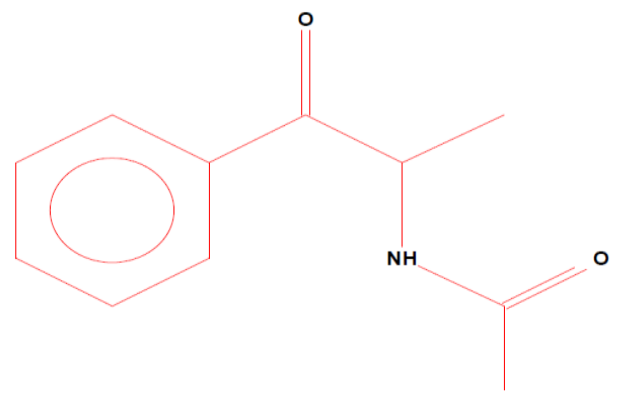

\begin{tabular}{|c|c|c|c|}
\hline Hith & Compound Name & M.W. & Formula \\
\hline 1 & SI.WCATHNNONE, NAACETYL. & 191 & C11H1302N \\
\hline 2 & ACETAMIDE, 22,2.TRRFLUUORO. & 113 & C2HLNF3 \\
\hline 3 & ACETAMIDE, 22,2.5TRFFLUORO. & 113 & CLH2ONF3 \\
\hline 4 & 2.3.PYPRIDNEDICARBOXYLLC ACID & 167 & C7H5OAN \\
\hline
\end{tabular}

19.27

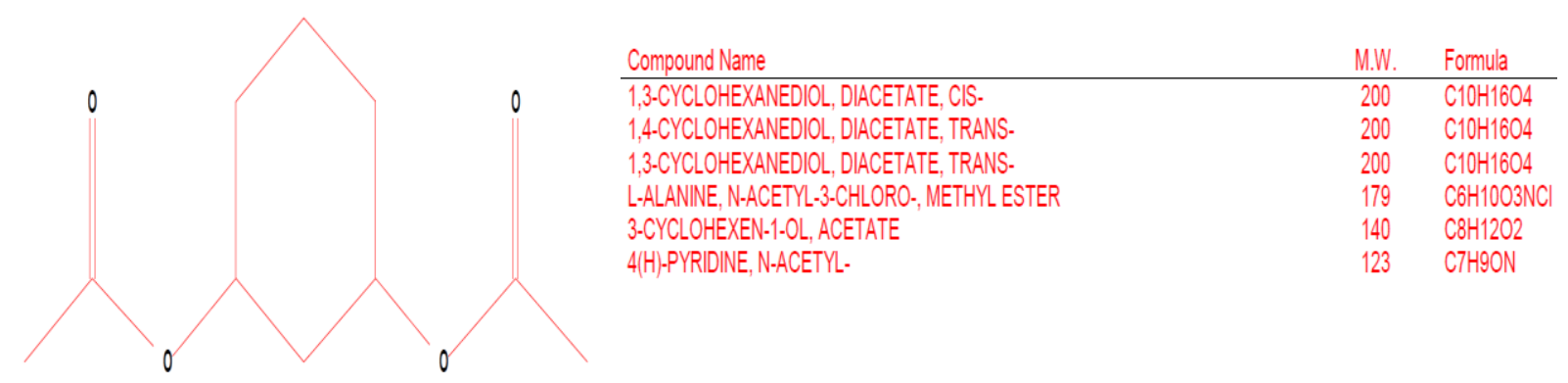


19.75

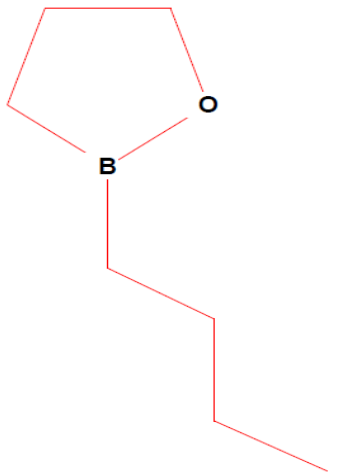

Compound Name

OXIOLOLANE

ETHER, HEXYL PENTYL

SULFUROUS ACID, ISOHEXYL 2-PENTYL ESTER

5,8-DOXXATETRADECANE

DI(TETRAHYDROFURFURYL)ADIPATE

PYRROLIDINE, 1,1-METHYLENEBIS-

2-ETHYL-1-BUTANOL, TRIFLUOROACETATE

SUCCINIC ACID HEXADECYL TETRAHYDROFURFURYL ESTER

-BUTANOL, 4-(HEXYLOXY)

1

2-ETHYLBUTYLISOBUTYRATE

SUCCINIC ACID HEPTADECYL TETRAHYDROFURFURYLESTER

1-OCTACOSANOL, 2,4,6.8-TETRAMETHYL-(ALL-R)-

SULFUROUS ACID, ISOHEXYL PENTYL ESTER

SULFUROUS ACID, NONYL 2-PROPYL ESTER

BUTANOIC ACID, 2-ETHYL-, BUTYL ESTER
DICHLOROACETIC ACID, 6-ETHYL-3-OCTYL ESTER

\begin{tabular}{cl} 
M.W. & Formula \\
\hline 126 & $\mathrm{C} 7 \mathrm{H} 15 \mathrm{OB}$ \\
172 & $\mathrm{C} 11 \mathrm{H} 24 \mathrm{O}$ \\
212 & $\mathrm{C} 8 \mathrm{H} 14 \mathrm{O} 2 \mathrm{Cl} 2$ \\
236 & $\mathrm{C} 11 \mathrm{H} 24 \mathrm{OS} \mathrm{S}$ \\
202 & $\mathrm{C} 12 \mathrm{H} 26 \mathrm{O} 2$ \\
143 & $\mathrm{C} 6 \mathrm{H} 9 \mathrm{O} 3 \mathrm{~N}$ \\
314 & $\mathrm{C} 16 \mathrm{H} 26 \mathrm{O} 6$ \\
154 & $\mathrm{C} 9 \mathrm{H} 18 \mathrm{~N} 2$ \\
198 & $\mathrm{C} 8 \mathrm{H} 13 \mathrm{O} 2 \mathrm{~F} 3$ \\
236 & $\mathrm{C} 11 \mathrm{H} 24 \mathrm{O} 3$ \\
426 & $\mathrm{C} 25 \mathrm{H} 46 \mathrm{~S}$ \\
174 & $\mathrm{C} 10 \mathrm{H} 22 \mathrm{O} 2$ \\
454 & $\mathrm{C} 27 \mathrm{H} 50 \mathrm{O} 5$ \\
172 & $\mathrm{C} 10 \mathrm{H} 20 \mathrm{O} 2$ \\
440 & $\mathrm{C} 26 \mathrm{H} 4805$ \\
466 & $\mathrm{C} 32 \mathrm{H} 660$ \\
236 & $\mathrm{C} 11 \mathrm{H} 24 \mathrm{OS}$ \\
250 & $\mathrm{C} 12 \mathrm{H} 26 \mathrm{O} S$ \\
172 & $\mathrm{C} 10 \mathrm{H} 20 \mathrm{~S} 2$ \\
268 & $\mathrm{C} 12 \mathrm{H} 22 \mathrm{O} 2 \mathrm{Cl} 2$
\end{tabular}

20.511

21.23

22.29
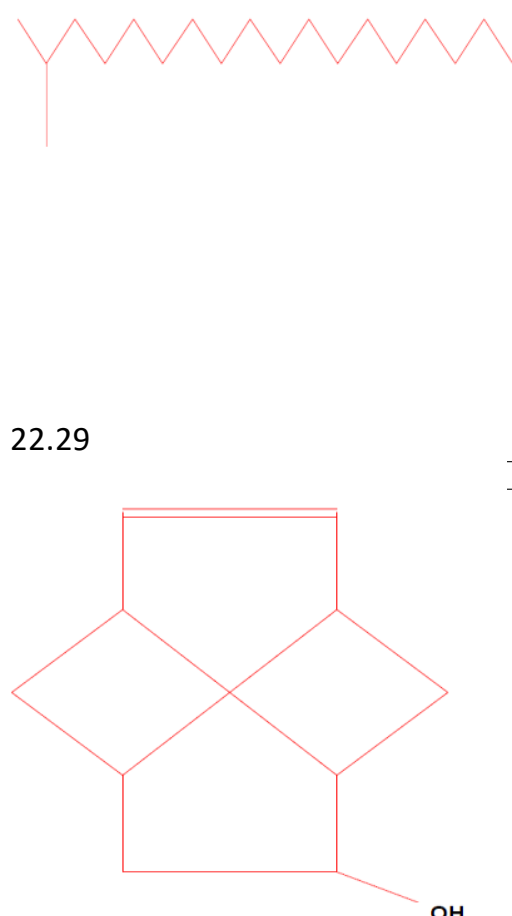

\begin{tabular}{|c|c|c|c|}
\hline Hit & Compound Name & M.W. & Formula \\
\hline 1 & 2,5-DIAMINO-2-METHYLPENTANOIC ACID & 146 & $\mathrm{C} 6 \mathrm{H} 14 \mathrm{O} 2 \mathrm{~N} 2$ \\
\hline 2 & 4-CYCLOOCTEN-1-AMINE, N-METHYL- & 139 & $\mathrm{C} 9 \mathrm{H} 17 \mathrm{~N}$ \\
\hline 3 & 2-T-BUTYLPEROXY-2-ETHYLBUTAN-1-OL, BUTYRATE ESTER & 260 & $\mathrm{C} 14 \mathrm{H} 28 \mathrm{O} 4$ \\
\hline 4 & OCTANE, 1,1'-OXYBIS- & 242 & $\mathrm{C} 16 \mathrm{H} 340$ \\
\hline & OCTANE, 1,1'-OXYBIS- & 242 & $\mathrm{C} 16 \mathrm{H} 340$ \\
\hline 6 & METHOXYACETIC ACID, OCTYL ESTER & 202 & $\mathrm{C} 11 \mathrm{H} 22 \mathrm{O} 3$ \\
\hline 7 & ACETIC ACID, TRICHLORO-, OCTYL ESTER & 274 & $\mathrm{C} 10 \mathrm{H} 17 \mathrm{O} 2 \mathrm{Cl} 3$ \\
\hline 8 & 10-UNDECENOIC ACID, OCTYL ESTER & 296 & $\mathrm{C} 19 \mathrm{H} 36 \mathrm{O} 2$ \\
\hline & BUTANOIC ACID, OCTYL ESTER & 200 & $\mathrm{C} 12 \mathrm{H} 24 \mathrm{O} 2$ \\
\hline 10 & 2- BROMOPROPIONIC ACID, OCTYL ESTER & 264 & $\mathrm{C} 11 \mathrm{H} 21 \mathrm{O} 2 \mathrm{Br}$ \\
\hline 11 & 5-OCTADECENAL & 266 & $\mathrm{C} 18 \mathrm{H} 34 \mathrm{O}$ \\
\hline 12 & 4-AMINO-6-METHYL-PIPERIDIN-2-ONE & 128 & $\mathrm{C} 6 \mathrm{H} 12 \mathrm{ON} 2$ \\
\hline 13 & CHLOROMETHYL OCTYL ETHER & 178 & $\mathrm{C} 9 \mathrm{H} 19 \mathrm{OCl}$ \\
\hline 14 & 2,4(1H,3H)-PYRIDINEDIONE, 1-BETA.-D-RIBOFURANOSYL- & 243 & $\mathrm{C} 10 \mathrm{H} 1306 \mathrm{~N}$ \\
\hline 15 & SULFUROUS ACID, DECYL 2-PROPYL ESTER & 264 & $\mathrm{C} 13 \mathrm{H} 28 \mathrm{O} 3 \mathrm{~S}$ \\
\hline 16 & HEXYL OCTYL ETHER & 214 & $\mathrm{C} 14 \mathrm{H} 300$ \\
\hline 17 & 2-PROPENOIC ACID, PENTADECYL ESTER & 282 & $\mathrm{C} 18 \mathrm{H} 34 \mathrm{O} 2$ \\
\hline 18 & $1,2,3$-CYCLOHEXANETRIOL & 132 & $\mathrm{C} 6 \mathrm{H} 12 \mathrm{O} 3$ \\
\hline 19 & 2-PROPENOIC ACID, TRIDECYL ESTER & 254 & $\mathrm{C} 16 \mathrm{H} 30 \mathrm{O} 2$ \\
\hline
\end{tabular}

2-PROPENOIC ACID, TRIDECYL ESTER

$\mathrm{C} 16 \mathrm{H} 30 \mathrm{O} 2$

\begin{tabular}{|c|c|c|c|}
\hline Hit & Compound Name & M.W. & Formula \\
\hline 1 & OCTADECANE, 2-METHYL- & 268 & $\mathrm{C} 19 \mathrm{H} 40$ \\
\hline 2 & HEXADECANE & 226 & $\mathrm{C} 16 \mathrm{H} 34$ \\
\hline 3 & NONADECANE, 2-METHYL- & 282 & $\mathrm{C} 2 \mathrm{OH} 42$ \\
\hline 4 & EICOSANE, 2-METHYL- & 296 & $\mathrm{C} 21 \mathrm{H} 44$ \\
\hline 5 & TRICOSANE, 2-METHYL- & 338 & $\mathrm{C} 24 \mathrm{H} 50$ \\
\hline 6 & TRICOSANE, 2-METHYL- & 338 & $\mathrm{C} 24 \mathrm{H} 50$ \\
\hline 7 & DOCOSANE, 2,21-DIMETHYL- & 338 & $\mathrm{C} 24 \mathrm{H} 50$ \\
\hline 8 & HEPTADECANE, 2,6,10,14-TETRAMETHYL- & 296 & $\mathrm{C} 21 \mathrm{H} 44$ \\
\hline 9 & HEXADECANE & 226 & $\mathrm{C} 16 \mathrm{H} 34$ \\
\hline 10 & 1-IODO-2-METHYLUNDECANE & 296 & $\mathrm{C} 12 \mathrm{H} 25 \mathrm{I}$ \\
\hline 11 & PENTADECANE & 212 & $\mathrm{C} 15 \mathrm{H} 32$ \\
\hline 12 & NONADECANE, 2-METHYL- & 282 & $\mathrm{C} 20 \mathrm{H} 42$ \\
\hline 13 & UNDECANE, 2,10-DIMETHYL- & 184 & $\mathrm{C} 13 \mathrm{H} 28$ \\
\hline 14 & HEPTADECANE, 2-METHYL- & 254 & $\mathrm{C} 18 \mathrm{H} 38$ \\
\hline 15 & HEXADECANE & 226 & $\mathrm{C} 16 \mathrm{H} 34$ \\
\hline 16 & OCTADECANE, 1-IODO- & 380 & $\mathrm{C} 18 \mathrm{H} 37 \mathrm{I}$ \\
\hline 17 & TETRADECANE & 198 & $\mathrm{C} 14 \mathrm{H} 30$ \\
\hline 18 & HEPTADECANE, 4-METHYL- & 254 & $\mathrm{C} 18 \mathrm{H} 38$ \\
\hline 19 & PENTADECANE, 2-METHYL- & 226 & $\mathrm{C} 16 \mathrm{H} 34$ \\
\hline 20 & DECANE, 2,6,8-TRIMETHYL- & 184 & $\mathrm{C} 13 \mathrm{H} 28$ \\
\hline
\end{tabular}

190

\begin{tabular}{|c|c|c|c|}
\hline Hit & Compound Name & M.W. & Formula \\
\hline 1 & TRICYCLO[4.4.0.0(3,8)]DEC-9-EN-4-OL & 150 & $\mathrm{C} 10 \mathrm{H} 140$ \\
\hline 2 & 2,4-DECADIEN-1-OL, (E,Z)- & 154 & $\mathrm{C} 10 \mathrm{H} 180$ \\
\hline 3 & 9,10-DIMETHYLENETRICYCLO[4.2.1.1(2,5)]DECANE & 160 & $\mathrm{C} 12 \mathrm{H} 16$ \\
\hline 4 & 1,2,4-METHENOCYCLOBUT[CD]INDEN-3(1H)-ONE, OCTAHYDRO- & 160 & $\mathrm{C} 11 \mathrm{H} 12 \mathrm{O}$ \\
\hline 5 & BENZENE, [(CYCLOHEX-1-EN-3-YL)METHYL]- & 172 & $\mathrm{C} 13 \mathrm{H} 16$ \\
\hline 6 & 4,7-METHANOISOBENZOFURAN-1,3-DIONE, 3A,4,7,7A-TETRAHYDRO-5-METHYL- & 178 & $\mathrm{C} 10 \mathrm{H} 1003$ \\
\hline 7 & 1-(2-METHYLENECYCLOHEXYL)-3-PHENYLPROPAN-1-OL & 230 & $\mathrm{C} 16 \mathrm{H} 22 \mathrm{O}$ \\
\hline 8 & 1,3,5-DODECATRIENE & 164 & $\mathrm{C} 12 \mathrm{H} 2 \mathrm{O}$ \\
\hline 9 & 6-[(1E)-1,3-BUTADIENYL]-1,4-CYCLOHEPTADIENE & 146 & $\mathrm{C} 11 \mathrm{H} 14$ \\
\hline 10 & 4,7-METHANOISOBENZOFURAN-1,3-DIONE, 3A,4,7,7A-TETRAHYDROMETHYL- & 178 & $\mathrm{C} 10 \mathrm{H} 1003$ \\
\hline 11 & 3-[(1Z)-1,3-BUTADIENYL]-4-VINYLCYCLOPENTENE & 146 & $\mathrm{C} 11 \mathrm{H} 14$ \\
\hline 12 & 6-[(1Z)-1,3-BUTADIENYL]-1,4-CYCLOHEPTADIENE & 146 & $\mathrm{C} 11 \mathrm{H} 14$ \\
\hline 13 & CYCLOBUTANE, 1-(1,3-BUTADIENYL)-2-VINYL- & 134 & $\mathrm{C} 10 \mathrm{H} 14$ \\
\hline 14 & 1,3,7,11-CYCLOTETRADECATETRAENE & 188 & $\mathrm{C} 14 \mathrm{H} 20$ \\
\hline 15 & CYCLOBUTANE, 1,2-BIS(1,3-BUTADIENYL)- & 160 & $\mathrm{C} 12 \mathrm{H} 16$ \\
\hline 16 & 1,2-BIS(3-CYCLOHEXENYL)ETHYLENE & 188 & $\mathrm{C} 14 \mathrm{H} 2 \mathrm{O}$ \\
\hline 17 & 2,7-METHANONAPHTHALENE, 1,2,4A,7,8,8A-HEXAHYDRO- & 146 & $\mathrm{C} 11 \mathrm{H} 14$ \\
\hline 18 & 1,4,4A,5,8,8A-HEXAHYDRO-NAPHTHALENE & 134 & $\mathrm{C} 10 \mathrm{H} 14$ \\
\hline 19 & $1,2,4 \mathrm{~A}, 4 \mathrm{~B}, 7,8,8 \mathrm{~A}, 8 \mathrm{~B}-\mathrm{OCT}$ AHYDROBIPHENYLENE & 160 & $\mathrm{C} 12 \mathrm{H} 16$ \\
\hline 20 & TRICYCLO[4.3.0.0(3,7)]NON-8-EN-4-OL & 136 & $\mathrm{C} 9 \mathrm{H} 12 \mathrm{O}$ \\
\hline
\end{tabular}


23.08

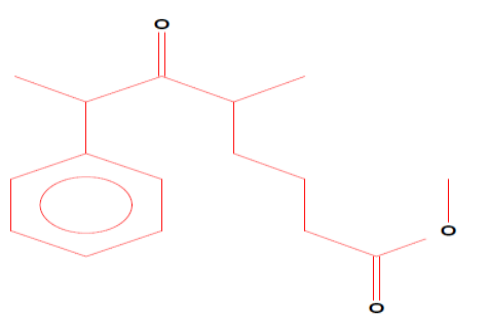

\begin{tabular}{|c|c|c|c|}
\hline & Compound Name & M.W. & Formula \\
\hline & METHYL 5-(2-PHENYLPROPIONYL)HEXANOATE & 262 & $\mathrm{C} 16 \mathrm{H} 22 \mathrm{O} 3$ \\
\hline & $\begin{array}{l}\text { 2-METHYL-1-PHENYL-2-PROPEN-1-OL } \\
\text { TRICYCIOL }\end{array}$ & 148 & $\begin{array}{l}\mathrm{C} 1 \mathrm{OH} 12 \mathrm{O} \\
\mathrm{C} 11 \mathrm{H} 12 \mathrm{O}\end{array}$ \\
\hline
\end{tabular}

26.54

30.58

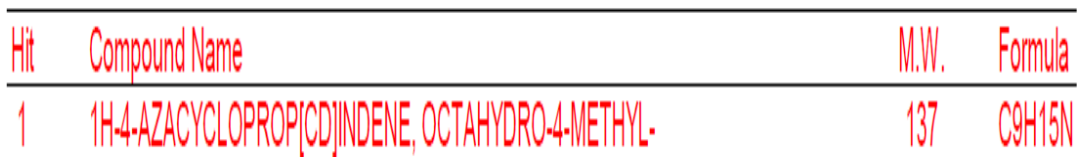

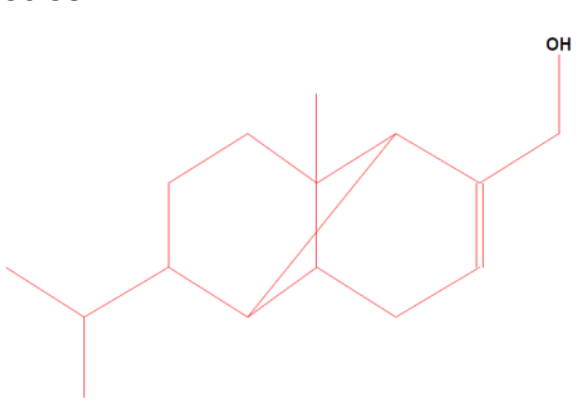

\begin{tabular}{clll} 
Hit & Compound Name & M.W. & Formula \\
\hline 1 & TRICYCLO[4.4.0.0(2,7)]DEC-3-ENE-3-METHANOL, 1-METHYL-8-(1-METHYLETHYL)- & 220 & $\mathrm{C} 15 \mathrm{H} 240$ \\
2 & $2,6-D I M E T H Y L-4-N I T R O-3-P H E N Y L-C Y C L O H E X A N O N E$ & 247 & $\mathrm{C} 14 \mathrm{H} 1703 \mathrm{~N}$ \\
3 & $2,5-0$-OCTADECADIYNOIC ACID, METHYL ESTER & 290 & $\mathrm{C} 19 \mathrm{H} 3002$ \\
4 & 10,12-DOCASADIYNDIOIC ACID & 362 & $\mathrm{C} 22 \mathrm{H} 3404$ \\
5 & FALCARINOL & 244 & $\mathrm{C} 17 \mathrm{H} 240$ \\
6 & 10-HENEICOSENE, 11-PHENYL- & 370 & $\mathrm{C} 27 \mathrm{H} 46$ \\
7 & $4,7-0$ CTADECADIYNOIC ACID, METHYL ESTER & 290 & $\mathrm{C} 19 \mathrm{H} 3002$ \\
8 & METHYL 5,7-HEXADECADIYNOATE & 262 & $\mathrm{C} 17 \mathrm{H} 2602$ \\
9 & ESTRA-5(10)-EN-3-ONE-17-OL,ACETATE & 316 & $\mathrm{C} 2 \mathrm{OH} 2803$ \\
10 & (4,4-DIMETHYL-2,4,5,6-TETRAHYDRO-1H-INDEN-2-YL)ACETIC ACID & 206 & $\mathrm{C} 13 \mathrm{H} 1802$ \\
11 & METHYL 8,10-OCTADECADIYNOATE & 290 & $\mathrm{C} 19 \mathrm{H} 3002$ \\
12 & METHYL 7,9-OCTADECADIYNOATE & 290 & $\mathrm{C} 19 \mathrm{H} 3002$ \\
13 & METHYL OCTADEC-6,9-DIEN-12-YNOATE & 290 & $\mathrm{C} 19 \mathrm{H} 3002$ \\
14 & 1-(3,3-DIMETHYL-1-YL)-2,2-DIMETHYLCYCLOPROPENE-3-CARBOXYLIC ACID & 192 & $\mathrm{C} 12 \mathrm{H} 1602$
\end{tabular}

Figure.7 Showing Phyto-chemicals identified in the Ethyl acetate extract of the Vitis vinifera peel by GC-MS

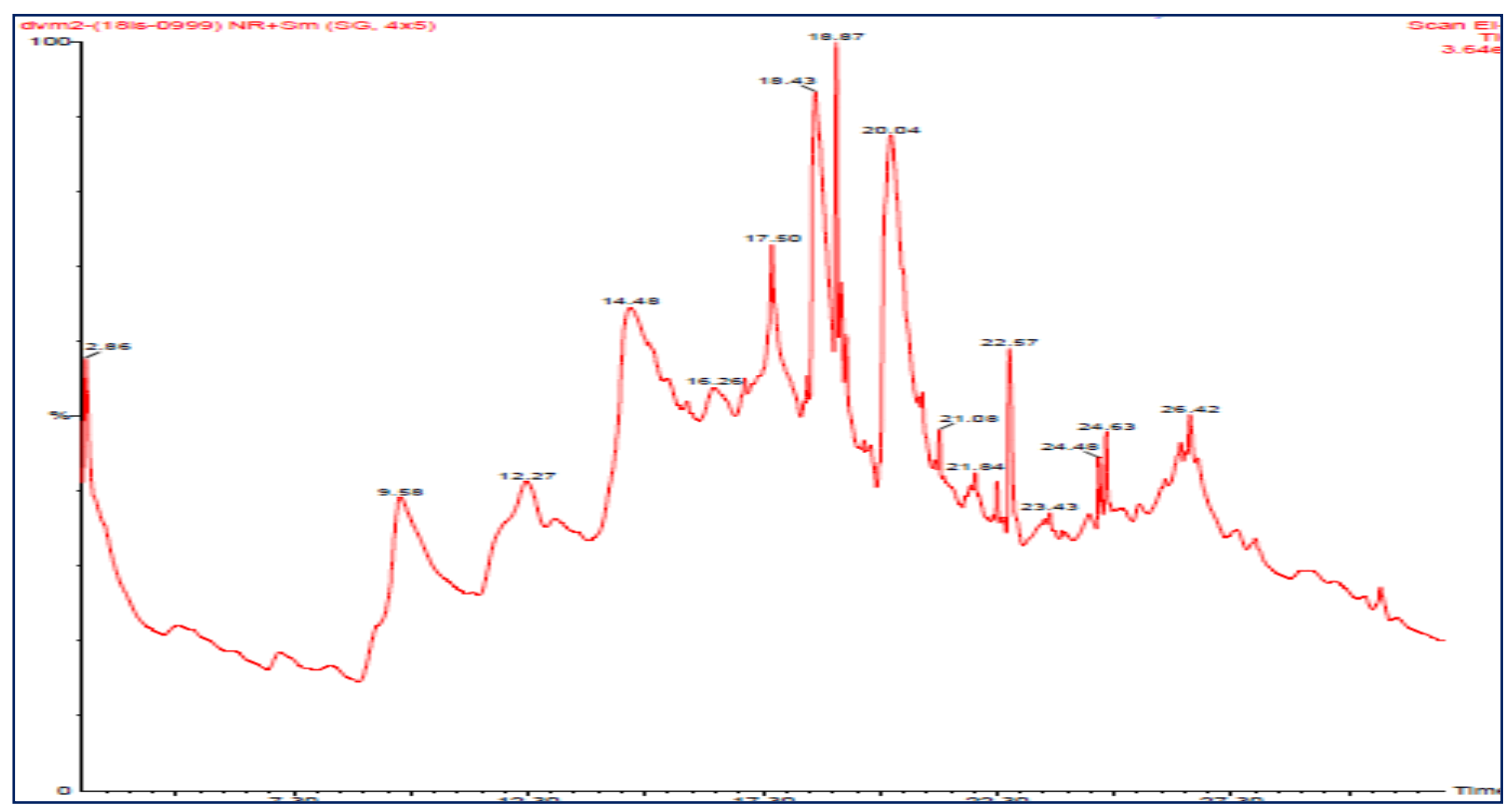

Figure.8 Showing GC-MS Chromatogram of methanol extract of the Vitis vinifera peel 
9.58

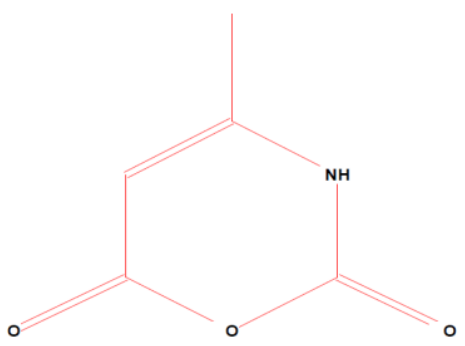

12.27

HO

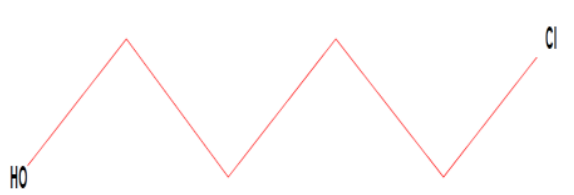

Compound Name

4-METHYL-1,3-OXAZINE-2,6[3H]-DIONE

DEC-9-EN-6-OXO-1-YLAMIDE

1,2,5-OXADIAZOLE-3-CARBOHYDROXYMIC ACID, 4-AMINO

(1)-PYRIAZONE-3-CARBOHYROXYMIC ACID, 4-AMI

GLUTAMINE, N-METHYL-

GLUTAMINE, N-METHYL-

$3(2 \mathrm{H})$-ISOXAZOLONE, 4,5-DIMETHYL-

2,4(1H,3H)-PYRIMIDINEDIONE, 1-[3,5-DIHYDROXY-6-HYDROXYMETHYL]

1,2,3-TRIAZOLE-4-METHANOL, 1-(4-AMINOFURAZAN-3-YL)-

3-METHYL-3,5--(CYANOETHYL) TETRAHYDRO-4-THIOPYRANONE

CYCLOHEPTANOIDIMIDAZOLIDINE 13-DIHYDROXY-2-METHYL-

1H-IMIDAZOLE, 2,4-DIMETHYL-

RONIDAZOLE

PROPANAL, 2METHYL- OXIME

L-GUANIDINOSUCCINIMIDE

5-AMINO-3,4-DIMETHYL-ISOXAZOLE

SPIRO[TETRAHYDROFURAN-3,5'-HYDANTOIN]

1,3-OXAZIN-2-ONE, TETRAHYDRO-3-METHYL-5,6-TRIMETHYLENE, CIS-

1 H-1,2,3-TRIAZOLE-5-METHANOL, 1-(4-AMINO-1,2,5-OXADIAZOL-3-YL)-

\begin{tabular}{|c|c|c|c|}
\hline Hit & Compound Name & M.W. & Formula \\
\hline 1 & 4-CHLORO-1-BUTANOL & 108 & $\mathrm{C} 4 \mathrm{H} 9 \mathrm{OCl}$ \\
\hline 2 & 4-CHLORO-1-BUTANOL & 108 & $\mathrm{C} 4 \mathrm{H} 9 \mathrm{OCl}$ \\
\hline${ }_{4}^{3}$ & $\begin{array}{l}\text { OXIRANE, PROOYL- } \\
\text { PROPYL ALDOXIME, 2-METHYL- ANTI- }\end{array}$ & $\begin{array}{l}86 \\
87\end{array}$ & $\begin{array}{l}\text { C5H10O } \\
\text { C4H9ON }\end{array}$ \\
\hline 5 & 3-ETHYL-3-METHYLDIAZIRIDINE & 86 & $\begin{array}{lll}C 4 H \\
C 410 N 2\end{array}$ \\
\hline 6 & 4-HYDROXYBUTYL ACRYLATE & 144 & $\mathrm{C} 7 \mathrm{H} 12 \mathrm{O} 3$ \\
\hline 7 & 4,5-DIHYDRO-2-METHYLIMIDAZOLE-4-ONE & 98 & C4H6ON2 \\
\hline 8 & 4-HYDROXYBUTYL ACRYLATE & 144 & $\mathrm{C} 7 \mathrm{H} 12 \mathrm{O} 3$ \\
\hline & 4-HYDROXYBUTYL ACRYLATE & 144 & $\mathrm{C} 7 \mathrm{H} 12 \mathrm{O} 3$ \\
\hline 10 & 1,2,5-TRIAZOLE 2-OXIDE, 1-SEC-BUTYL-3-NITRO-4-AMINO- & 201 & $\mathrm{C} 6 \mathrm{H} 1103 \mathrm{~N} 5$ \\
\hline 11 & 1-DIMETHYLAMINO-2-NITROETHYLENE & 116 & $\mathrm{C} 4 \mathrm{H} 8 \mathrm{O} 2 \mathrm{~N} 2$ \\
\hline 12 & L-GALA-L-IDO-OCTOSE & 240 & $\mathrm{C} 8 \mathrm{H} 1608$ \\
\hline 13 & 5-AMINO-3,4-DIMETHYL-ISOXAZOLE & 112 & $\mathrm{C} 5 \mathrm{H} 8 \mathrm{ON} 2$ \\
\hline 14 & PENTANOIC ACID, 3-HYDROXY-4-METHYL-, METHYL ESTER & 146 & $\mathrm{C} 7 \mathrm{H} 14 \mathrm{O} 3$ \\
\hline & CYCLOHEPTANOL & 114 & $\mathrm{C} 7 \mathrm{H} 14 \mathrm{O}$ \\
\hline $\begin{array}{l}16 \\
17\end{array}$ & $\begin{array}{l}\text { 3,8,9-TRIOXABICYCLO[-2.2.1]NONANE \# } \\
\text { ACETIC ACID PENTY ESTER }\end{array}$ & ${ }_{130}^{130}$ & $\begin{array}{l}\mathrm{C} 6 \mathrm{H} 10 \mathrm{OO} 3 \\
\mathrm{C} 7 \mathrm{H} 14 \mathrm{O} 2\end{array}$ \\
\hline 18 & CYCLOHEXANOL, 2-METHYL- TRANS- & 114 & $\mathrm{C} 7 \mathrm{H} 140$ \\
\hline 19 & ISOTHIOUREA, 2-(2-OCTYLSULFONYL)ETHYL- & 280 & $\mathrm{C} 11 \mathrm{H} 24 \mathrm{O} 2 \mathrm{~N} 2$ \\
\hline 20 & 4-AMINO-1-HEXANOL & 117 & $\mathrm{C} 6 \mathrm{H} 150 \mathrm{~N}$ \\
\hline
\end{tabular}

14.47

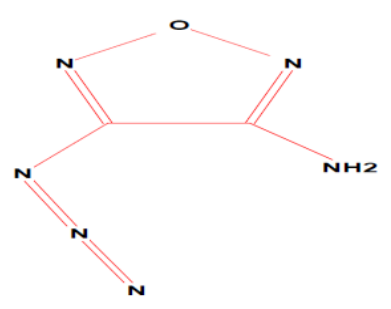

\begin{tabular}{clcl} 
Hit & Compound Name & M.W. & Formula \\
\hline 1 & FURAZANAMINE, 4-AZIDO- & 126 & C2H2ON6 \\
2 & 3-ETHYLAMINO-5-HEXENE-2-OL & 143 & C8H17ON
\end{tabular}

17.49
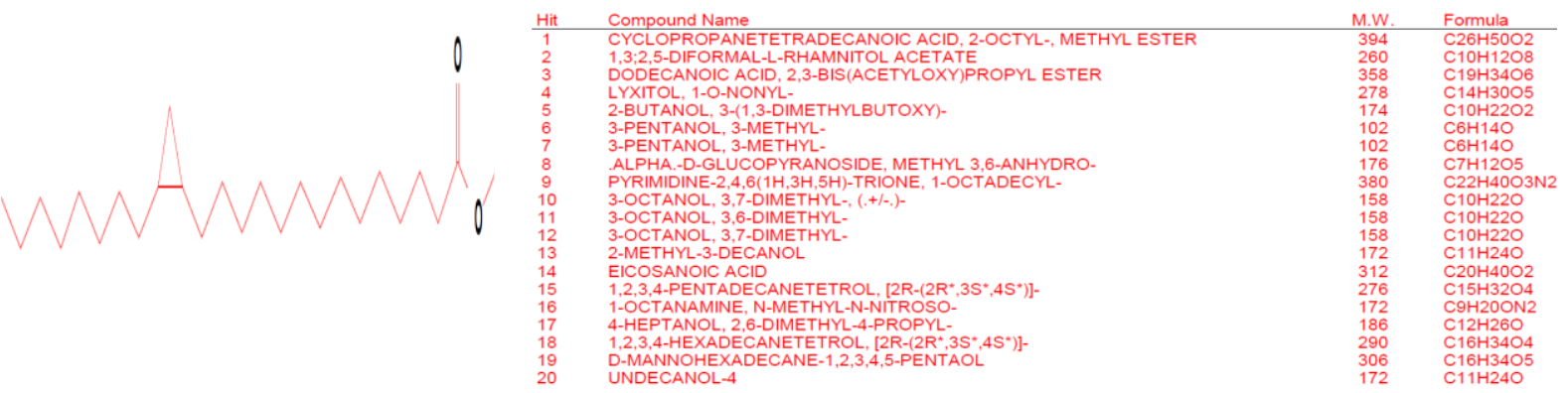

18.42

\begin{tabular}{|c|c|c|c|}
\hline Hit & Compound Name & M.W. & Formula \\
\hline 1 & 2-PENTENOIC ACID, 4-OXO-, METHYL ESTER, (Z)- & 128 & $\mathrm{C} 6 \mathrm{H} 8 \mathrm{O} 3$ \\
\hline 2 & PENTANOIC ACID, 3,5-DIOXO-, METHYL ESTER & 144 & $\mathrm{C} 6 \mathrm{H} 8 \mathrm{O} 4$ \\
\hline & HEXANOIC ACID, 3-OXO-, METHYL ESTER & 144 & $\mathrm{C} 7 \mathrm{H} 12 \mathrm{O} 3$ \\
\hline & 2-PENTENOIC ACID, 4-OXO-, METHYL ESTER, (E)- & & $\mathrm{C} 6 \mathrm{H} 8 \mathrm{O} 3$ \\
\hline & 2(5H)-FURANONE, 5,5-DIMETHYL- & & $\mathrm{C} 6 \mathrm{H} 8 \mathrm{O} 2$ \\
\hline & 1,3-DIOXOLANE, 2,2-DIMETHYL-4-HYDROXYMETHYL-5-(2-HYDROXYPROPYL)- & & $\mathrm{C} 9 \mathrm{H} 18 \mathrm{O} 4$ \\
\hline & XYLOPYRANOSIDE, METHYL 4-THIO-, TRIACETATE, .ALPHA.-D- & & $\mathrm{C} 12 \mathrm{H} 1807 \mathrm{~S}$ \\
\hline & 2(5H)-FURANONE, 5,5-DIMETHYL- & 112 & $\mathrm{C} 6 \mathrm{H} 8 \mathrm{O} 2$ \\
\hline & HEPTANEDIOIC ACID, 4-METHYL-, DIMETHYL ESTER & & $\mathrm{C} 10 \mathrm{H} 1804$ \\
\hline & $\begin{array}{l}\text { ACETIC ACID, 2-METHYL-6-OXO-HEPTYL ESTER } \\
\text { HEXANOIC ACID 5-OXO-METHYL ESTER }\end{array}$ & & $\mathrm{C} 10 \mathrm{H} 18 \mathrm{O} 3$ \\
\hline & $\begin{array}{l}\text { HEXANOIC ACID, 5-OXO-, METHYL ESTER } \\
\text { HEPTANEDIOICACID, 3-METHYL- DIMETHYL ESTER }\end{array}$ & & $\mathrm{C} 7 \mathrm{H} 12 \mathrm{O} 3$ \\
\hline 13 & $\begin{array}{l}\text { HEPTANEDIOIC ACID, 3-METHYL-, DIMETHYL ESTER } \\
\text { METHYL 4-OXO-2-PENTENOATE }\end{array}$ & 202 & $\begin{array}{l}\mathrm{C} 10 \mathrm{H} 18 \mathrm{O} 4 \\
\mathrm{C} 6 \mathrm{H} 8 \mathrm{O} 3\end{array}$ \\
\hline 14 & 5-ISOXAZOLIDINECARBOXYLIC ACID, 5-METHYL-2-[5-O-METHYL-2,3-O-(1-METHY & 331 & $\mathrm{C} 15 \mathrm{H} 2507 \mathrm{~N}$ \\
\hline & 2-PROPANOL, 1-(1,3-DIMETHYLBUTOXY)- & & $\mathrm{C} 9 \mathrm{H} 20 \mathrm{O} 2$ \\
\hline & 3-ETHYL-4-METHYL-3-HEPTANOL & 158 & $\mathrm{C} 10 \mathrm{H} 22 \mathrm{O}$ \\
\hline & 2-FURANHEXANOIC ACID, TETRAHYDRO-.BETA.,.DELTA.-DIHYDROXY-5-METHO & & $\mathrm{C} 12 \mathrm{H} 22 \mathrm{O} 6$ \\
\hline 18 & 5-ISOXAZOLIDINECARBOXYLIC ACID, 5-METHYL-2-[5-O-METHYL-2,3-O-(1-METHY & & $\mathrm{C} 15 \mathrm{H} 2507 \mathrm{~N}$ \\
\hline $\begin{array}{l}19 \\
20\end{array}$ & $\begin{array}{l}\text { BETA.L-ARAABNOPYRANOSSDE, METHYL 2-O-METHYL-3,4-O-(1-METHYLETHYLI } \\
\text { 14-DI-O-ACETYL-2,5-DI-O-METHYL-36-DIDEOXY-D-GLUCITOL }\end{array}$ & $\begin{array}{l}218 \\
262\end{array}$ & $\begin{array}{l}\mathrm{C} 10 \mathrm{H} 1805 \\
\mathrm{C} 12 \mathrm{H} 22 \mathrm{O} 6\end{array}$ \\
\hline
\end{tabular}


18.87

20.04

\begin{tabular}{clcl} 
Hit & Compound Name & M.W. & Formula \\
\hline 1 & 5-HEXENOIC ACID, METHYL ESTER & 128 & $\mathrm{C} 7 \mathrm{H} 12 \mathrm{O} 2$ \\
2 & 3-DODECEN-1-OL & 184 & $\mathrm{C} 12 \mathrm{H} 24 \mathrm{O}$ \\
3 & 2-HEPTADECENAL & 252 & $\mathrm{C} 17 \mathrm{H} 32 \mathrm{O}$ \\
4 & 4-OCTADECENOIC ACID, METHYL ESTER & 296 & $\mathrm{C} 19 \mathrm{H} 36 \mathrm{O} 2$ \\
5 & UNDECANENITRILE & 167 & $\mathrm{C} 11 \mathrm{H} 21 \mathrm{~N}$ \\
6 & 4-CHLORO-3-N-HEXYLTETRAHYDROPYRAN & 204 & $\mathrm{C} 11 \mathrm{H} 210 \mathrm{C}$ \\
7 & HEXANENITRILE, 5-METHYL- & 111 & $\mathrm{C} 7 \mathrm{H} 13 \mathrm{~N}$ \\
8 & DECANENITRLE & 153 & $\mathrm{C} 10 \mathrm{H} 19 \mathrm{~N}$ \\
9 & METHYL 3-CYCLOPROPYLPROPANOATE & 128 & $\mathrm{C} 7 \mathrm{H} 12 \mathrm{O} 2$ \\
10 & NONANENITRILE & 139 & $\mathrm{C} 9 \mathrm{H} 17 \mathrm{~N}$ \\
11 & E-2-OCTADECADECEN-1-OL & 268 & $\mathrm{C} 18 \mathrm{H} 36 \mathrm{O}$ \\
12 & UNDECANENITRILE & 167 & $\mathrm{C} 11 \mathrm{H} 21 \mathrm{~N}$ \\
13 & Z-3-OCTADECEN-1-OL ACETATE & 310 & $\mathrm{C} 20 \mathrm{H} 38 \mathrm{O} 2$ \\
14 & 5-HEXENOIC ACID, METHYL ESTER & 128 & $\mathrm{C} 7 \mathrm{H} 12 \mathrm{O} 2$ \\
15 & 1,19-EICOSADIENE & 278 & $\mathrm{C} 20 \mathrm{H} 38$ \\
16 & Z-2-OCTADECEN-1-OL ACETATE & 310 & $\mathrm{C} 20 \mathrm{H} 3802$ \\
17 & DECANENITRILE & 153 & $\mathrm{C} 10 \mathrm{H} 19 \mathrm{~N}$ \\
18 & CYCLOHEXANOL, 2-METHYL-, ACETATE, CIS- & 156 & $\mathrm{C} 9 \mathrm{H} 16 \mathrm{O} 2$ \\
19 & OXALIC ACID, CYCLOHEXYLMETHYL PROPYL ESTER & 228 & $\mathrm{C} 12 \mathrm{H} 200 \mathrm{O}$ \\
20 & 2-DODECEN-1-OL & 184 & $\mathrm{C} 12 \mathrm{H} 240$
\end{tabular}

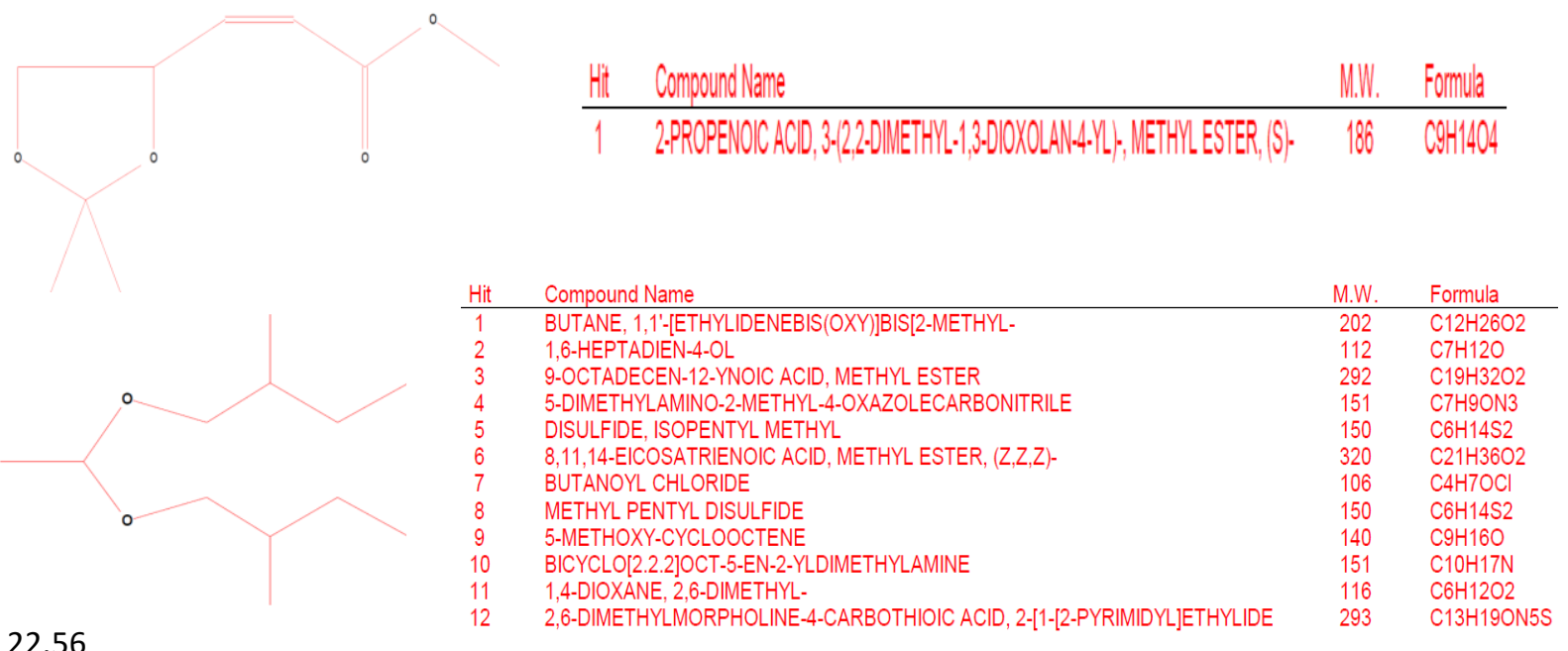

24.63

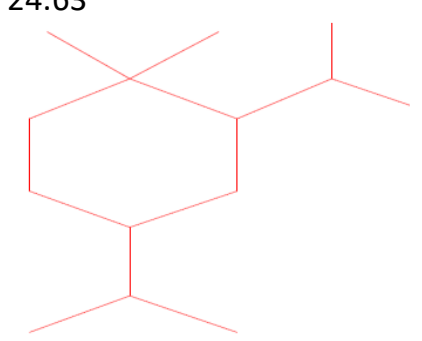

\begin{tabular}{|c|c|c|c|}
\hline $\mathbb{1}$ & Compound Name & M.W. & Formula \\
\hline & CYCLOHEXXNE, 2.4DDISOPROPYL-1,IDIMETHYL- & 196 & C144-28 \\
\hline & CYCLOHEXANE, 1,5DDISOPROPYL-23.JDIMETHYL- & 196 & C14428 \\
\hline & CYCLOHEXANE, 1,'-1.-METHYLPROPYLDENEEBIS. & 222 & C16H30 \\
\hline & OXALCCACID, HEPTADECYL 1-MENTHYL ESTER & 466 & C29H5404 \\
\hline & OXALLC ACDD, 1-MENTHYL PENTADECYL ESTER & 438 & C27H50O4 \\
\hline
\end{tabular}

26.41

\begin{tabular}{|c|c|c|c|}
\hline Hit & Compound Name & M.W. & Formula \\
\hline 1 & 1-HEXANOL,2-(HYDROXYMETHYL). & 132 & C7H1602 \\
\hline 2 & AMINOPROPIONAMIDE, N-METHYL-N-A-(1.PYRROLIDNYLL)-2BUTYYYLL-N'T-TBUT & 323 & C17H2903N3 \\
\hline 3 & 2.OCTENAL, (E). & 126 & C8H14O \\
\hline 4 & 3-METHYL-4.PHENYLTHIO)-2-PROP-2-ENYL-2,5-DIHYDROTHIOPHENE 1,1-DIOXI & 280 & C14H1602S2 \\
\hline 5 & FORMAMIDE, N-METHYL-N-A-[1-PYRROLLDNYL)-2-BUTYNYL]- & 180 & C10H160N2 \\
\hline
\end{tabular}

Figure.9 Showing Phyto-chemicals identified in the methanol extract of the Vitis vinifera peel by GC-MS 


\title{
Vitis vinifera Peel
}

\section{GC-MS analysis of Aqueous extract, Methanol and Ethyl acetate (Chromatogram and Phyto-chemical compounds Identification)}

\author{
5 \\ Synthesis of AgNPs by Aqueous extract of Vitis vinifera peel

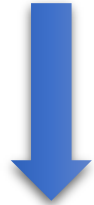 \\ Characterization studies by using UV-Visible spectrum \\ Dynamic light scattering analysis. \\ Scanning electron microscopy
}

\section{Flow chart of the Study}

\section{CONCLUSION:}

In the present study more than twenty-five constituents have been identified from Aqueous, Ethyl acetate and methanolic extracts of Vitis vinifera peel by GC-MS analysis. The presence of various bioactive compounds justifies their use for various ailments by traditional practitioners Xylose and Cyclohexane were present. However, further studies are undertaken to identify the mechanisms of these compounds and their potential applications for the diseases in the field of Pharmaceuticals because the peel is having the medical applications (Phytochemical and bio-active compounds) for health benefits.

\section{ACKNOWLEDGEMENT:}

Authors are thankful to Acharya N G Ranga Agricultural University, Nanotechnology laboratory, Tirupati for the carrying out this works.

\section{REFERENCES:}

[1] Gruenwald J., Brendler B.A. and Jaenicke C., PDR for herbalmedicines, $3 \mathrm{Rd}$ ed., Thomson PDR: Montvale, NJ. (2004).

[2] Anjaria J, Parabia M., Bhatt G., and Khamar R., Nature heals a glossary of selected indigenous medicinal plants of India. Sristi Innovations, Ahmedabad, India, (2002).

[3] Sriram K., Benkovic SA., Hebert MA., Miller DB., O'Callaghan JP., Induction of gp130-related cytokines and activation of JAK2/STAT3 pathway in astrocytes precedes upregulation of glial fibrillary acidic protein in the 1-methyl-4-phenyl-, 2, 3, 6-tetrahydropyridine model of neurodegeneration: Key signaling pathway for astrogliosis in vivo. J Biol Chem, 279:19936-9947, (2004)

[4] Balu M., Sangeetha P., Haripriya D., Panneerselvam C., Rejuvenation of antioxidant system in central nervous system of aged rats by grape seed extract. Neurosci. Lett, 383: 295-300, (2005).

[5] Aslanian A., Dizaji AA., Fahoomand P., Shahrary HA., Maheri N., Rouhnavaz S., Characterization of the nutritive value and protein fractions the Cornell net carbohydrateotien system in white and red grape (Vitis vinivera sp.) pomace. Research journal of biological sciences, 6(7): 298 - 303, (2011).

[6] Cetin ES., Altinoz D., Tarcan E., Baydar NG., Chemical composition of grape canes. Industrial Crops and Products, 34(1): 994 - 998, (2011).

[7] Vasil Georgiev., Anthony Ananga., Violeta Tsolova., Recent Advances and Uses of Grape Flavonoids as Nutraceuticals. Nutrients, 6: 391-415, (2014).

[8] Syed Zameer Hussain., Khushnuma Maqbool., GCMS: Principle, Technique and its application in Food Science; Division of Post-Harvest Technology. Int J Curr Sci, 13: 116 - 126, (2014).

[9] Dulundu E., Ozel Y., Topaloglu U., Grape seed extract reduces oxidative stress and fibrosis in experimental biliary obstruction. J. Gastroenterol Hepatol, 22:885892, (2007).

[10] Divya R., Supraja N., David E., Synthesis and Characterization of AgNPs from Vitis Vinifera Peel Extract and Its Antimicrobial Efficacy. Research and Development in material sciences, 9(3): ISSN: 25768840, (2019) 
[11] Supraja N., Kishore B., Rajasekhar KK., Padmavathamma M., Synthesis of Carica papaya (Leaf, peel and seed) extracts mediated $\mathrm{Ag}$ nanoparticles for industrial and medical applications. Chem.sci.eng.res, 2(5): 29-39, (2020)

[12] Divya R., Supraja N., David E., Synthesis and Characterization of ZnONPs from Vitis Vinifera Peel Extract and Its Antimicrobial Efficacy. Advancements in Bioequivalence and bioavailability, 2(2): ISSN: 26409275, (2019)

[13] Naresh S., Sunil KS., Akki Suma, Ashika BD., Chitrali Laha Roy., Dr. Balasubramanian Sathyamurthy., GCMS and FTIR analysis on the methanolic extract of red Vitis vinifera pulp. wjpls, 4(8): 153-159, (2018)

[14] Anonymous, Duke's phytochemical and ethnobotanical database. http://www.ars-grin.gov/ cgi-bin/duke/ethnobot.pl. (2014)

[15] Adewole E., Adewumi DF, Jonathan J., Fadaka AO., Phytochemical constituents and proximate analysis of orange peel (Citrus fruit). J. Adv. Bot. Zool., 1: 1-2, (2014).

[16] Supraja N., Prasad TNVKV., Gandhi AD., Anbumani D., Kavitha P., Babujanarthanam R., Synthesis, Characterization and Evaluation of Antimicrobia Efficacy and Brine Shrimp Lethality Assay of Alstonia Scholaris Stem Bark Extract Mediated ZnONPs. Biochem. Biophys. Rep, 14: 69-77, (2018).

[17] Supraja N., Prasad TNVKV., Soundariya M., Babujanarthanam R., Synthesis, characterization, and dose dependent antimicrobial and anticancerous activity of phycogenic silver nanoparticles against human hepatic carcinoma (HepG2) cell line. AIMS Bioengineering, 3 (4): 425-440, (2016)

[18] Supraja N., Avinash B., Prasad TNVKV. Nelumbo Nucifera Extracts Mediated Synthesis of Silver Nanoparticles for the Potential Applications in Medicine and Environmental Remediation. Adv. Nano Res, 5: 373 (2017).

[19] Uraku Anayo Joseph., Uraku Oluchi Helen., Nweke Friday Nwalo., Orji Obasi Uche., Igwenyi Ikechukwu Okorie., Edwin Nzubechukwu., Ezeani Nkiru Nwamaka., Phytochemical and GC-MS Evaluation of Bioactive Principle of Vitis vinifera Peels. Asian Journal of Applied Sciences, ISSN 1996-3343, DOI: 10.3923/ajaps.2018.192.198, (2018)

[20] Kadhim MJ., Al-Rubaye AB., Hameed IH., Determination of bioactive compounds of methanolic extract of Vitis vinifera using GC-MS. Int. J. Toxicol. Pharmacol. Res, 9: 113-126, (2017).

[21] Kumar KA., Vijayalakshmi K., GC-MS analysis of phytochemical constituents in ethanolic extract of Punica granatum Peel and Vitis vinifera seeds. Int. J. Pharma Bio Sci, 2: B461-B468, (2011).

[22] Bupesh G., Vijayakumar T., Manivannan S., Beerammal M., Manikadan E., Shanthi P., Vijaya AA., Identification of secondary metabolites, antimicrobial and antioxidant activity of grapefruit (Vitis vinifera) skin extract. Diabetes Obesity Int, 1(1): (2016). 